\title{
NITSCHE-BASED MODELS FOR THE UNILATERAL CONTACT OF PLATES
}

\author{
Mathieu Fabre ${ }^{1}$, CÉdric Pozzolini ${ }^{1, *}$ and Yves Renard ${ }^{2}$
}

\begin{abstract}
This paper aims to present different Nitsche-based models for the unilateral contact of plate structures. Our analysis is based on the consideration of Nitsche's method on a 3D structure with kinematic assumptions of thin or thick plate theories. This approach is compared to that of Gustafsson, Stenberg and Videman which consists of Nitsche's method applied directly on a 2D plate model. To simplify the presentation, we focus on the contact of an elastic plate with a rigid obstacle. The different approaches are compared numerically in terms of reliability compared to the 3D elastic model.
\end{abstract}

Mathematics Subject Classification. 65N22.

Received April 3, 2020. Accepted August 25, 2020.

\section{INTRODUCTION AND KINEMATICS OF PLATE MODELS}

The weak enforcement of unilateral contact conditions has already been treated by several techniques, the main ones being the penalty method, mixed/mortar methods (with standard or augmented Lagrangians) and, more recently, Nitsche's method. The penalty method is the simplest one but it leads to a supplementary approximation (it is not consistent in a strong sense) and quality of the approximation is sensitive to the choice of the penalty coefficient (see $[8,10,12])$. Mixed methods introduce an additional variable which, in that case, represents the contact stress. The corresponding weak form is consistent and leads numerically to an optimal convergence rate whenever an inf-sup condition is fulfilled (see [35]), which can be a difficulty for instance with immersed boundaries or simply on the interface of several boundaries for multi-body contact. Augmented Lagrangian techniques can be used for numerical solution for instance with Uzawa techniques (see [33]).

Nitsche's method [29] allows to treat boundary or interface conditions in a weak sense. The extension to the unilateral contact condition is proposed in [9,27,36]. An advantage of Nitsche's method is to allow a consistent approximation without any additional unknown and without discrete inf-sup condition to satisfy. In an overview [12] it is presented a simple and systematic procedure to derive a family of Nitsche's formulations that have different symmetry properties and different degrees of dependency on the stabilization parameter and for various boundary and interface conditions in 3D contact problems. This family is indexed by the so-called Nitsche symmetric parameter. Let us mention that, in the context of the standard finite element method, the skew-symmetric formulation proves to be very robust with respect to the stabilization parameter (see [10]).

Keywords and phrases. Contact problem, variational formulation, numerical scheme, Nitsche method.

1 ESI Group, 70 Rue Robert, Lyon 69006, France.

2 Université de Lyon, CNRS, INSA-Lyon, ICJ UMR5208, LaMCoS UMR5259, Villeurbanne F-69621, France.

*Corresponding author: cedric.pozzolini@esi-group.com 
We refer to [18] for a more detailed review on a priori error estimates for contact problems in elasticity. The case of contact between two elastic bodies, still in small deformations framework, is addressed in $[10,13]$. In [11] a variant of an unbiased Nitsche's method is proposed for the contact between two elastic bodies without making any difference between master and slave contact surfaces, which is an advantage for treatment of self-contact or multi-body contact.

With regard to unilateral contact of thin structures, a Nitsche approximation of the unilateral contact between a Kirchhoff-Love plate and a rigid obstacle is presented in $[23,24]$. In these references, the origin of the proposed method is the Kirchhoff-Love plate model subject to a unilateral contact condition. It is shown that an approximation using Lagrange multipliers with an additional stabilization term leads to a Nitsche method by static condensation of the multiplier.

The approach we consider in this paper is different. We start from the three-dimensional problem on which we apply the unilateral contact condition using Nitsche's method, as in [10]. Then, we apply the kinematics of different models of thin and thick plates, which allows us to write different approximated models of the unilateral contact of plate structures.

Let us now introduce the notations used and the plate models that we will consider. A thick or a thin elastic plate is a flat structure one dimension of which, called thickness, is small or very small compared to the others. For this kind of structure, starting from a priori hypotheses on the expression of the displacement fields, a two-dimensional problem is usually derived from the three-dimensional elasticity formulation by means of integration in the thickness direction (see for instance [17]). The unknown variables are then described on the mid-plane of the plate.

In what follows, we use the canonical orthonormal basis $\left(e_{1}, e_{2}, e_{3}\right)$ for the coordinate space $\mathbb{R}^{3}$. Let $\Omega$ be an open, bounded, connected subset of the plane $\operatorname{Span}\left(e_{1}, e_{2}\right)$, with Lipschitz-continuous boundary which defines the mid-plane of the plate. Then, the plate in its stress-free reference configuration coincides with the domain

$$
\Omega^{\varepsilon}:=\Omega \times(-\varepsilon, \varepsilon)=\left\{\left(x_{1}, x_{2}, x_{3}\right) \in \mathbb{R}^{3} \mid\left(x_{1}, x_{2}\right) \in \Omega \quad \text { and } \quad x_{3} \in(-\varepsilon, \varepsilon)\right\},
$$

where $2 \varepsilon>0$ is called the thickness. Note that in reference configuration the mid-plane is in the plane $x_{3}=0$.

We define on the boundary of $\Omega^{\varepsilon}$ an outward unit normal vector $n$ and a unit tangential vector $t$ such that the pair $(n, t)$ defines a direct orthonormal coordinate system. We consider a plate made of an elastic, homogeneous and isotropic material, whose mechanical constants are its Young's modulus $E$ and its Poisson's ratio $\nu$, with, as usual, $E>0$ and $0 \leq \nu<\frac{1}{2}$. In addition, $\delta_{i j}$ is Kronecker's symbol and the summation convention over repeated indices is adopted, Greek indices varying in $\{1,2\}$ and Latin indices in $\{1,2,3\}$. In the following, $\partial_{i}$ stands for the partial derivative with respect to $x_{i}$ and the second derivatives are $\partial_{i j}^{2}:=\frac{\partial^{2}}{\partial x_{i} \partial x_{j}}$.

In general plate theory, it is assumed that a mid-surface plane can be used to represent the three-dimensional plate in two-dimensional form. We consider the following very common linear approximation of the threedimensional displacements for $x=\left(x_{1}, x_{2}, x_{3}\right) \in \Omega^{\varepsilon}$ :

$$
\left\{\begin{array}{l}
u_{1}\left(x_{1}, x_{2}, x_{3}\right)=\underline{u}_{1}\left(x_{1}, x_{2}\right)+x_{3} \theta_{1}\left(x_{1}, x_{2}\right), \\
u_{2}\left(x_{1}, x_{2}, x_{3}\right)=\underline{u}_{2}\left(x_{1}, x_{2}\right)+x_{3} \theta_{2}\left(x_{1}, x_{2}\right), \\
u_{3}\left(x_{1}, x_{2}, x_{3}\right)=\underline{u}_{3}\left(x_{1}, x_{2}\right)+x_{3} \theta_{3}\left(x_{1}, x_{2}\right) .
\end{array}\right.
$$

In these expressions, $\underline{u}_{1}$ and $\underline{u}_{2}$ are the membrane displacements of the mid-plane points, $\underline{u}_{3}$ is the deflection, while $\theta_{1}, \theta_{2}$ are the section rotations, and $\theta_{3}$ is the pinching. In the case of a homogeneous isotropic material, the variational plate model often splits into two independent problems: the first, called the membrane problem, deals only with membrane displacements, while the second, called the bending problem, concerns deflection and rotations. Note also that some enhanced models have been proposed with a quadratic approximation in the variable $x_{3}$ for the transverse displacement $u_{3}$ instead of a linear one (see $[7,25]$ for instance) especially when a significant pinching of the plate occurs. However, we limit ourselves to a linear approximation along $x_{3}$. 
In this paper, we consider two types of plates models. On the one hand, the classical Kirchhoff-Love and Mindlin-Reissner models which are based on an additional plane stress approximation assumption, and on the other hand some simple solid plate models which do not include this additional assumption.

The Kirchhoff-Love plate model is based on the plane stress approximation and the following kinematic assumptions which can be seen as a special case of (1.1):

$$
\left\{\begin{array}{l}
\theta_{1}=-\partial_{1} u_{3}, \\
\theta_{2}=-\partial_{2} u_{3}, \\
\theta_{3}=0
\end{array}\right.
$$

Therefore, deflection is the only unknown for the bending Kirchhoff-Love plate problem and this displacement is independent of membrane displacements (i.e. in-plane displacements of the mid-surface) in the homogeneous isotropic case.

In the Mindlin-Reissner theory, a plane stress approximation is also considered and the normal to the midsurface remains straight but not necessarily perpendicular to the mid-surface. Shear stress is no longer neglected. The kinematics is also a special case of (1.1) obtained by neglecting the pinch, assuming

$$
\theta_{3}=0 \text {. }
$$

Finally, we consider two simple plate models which are not based on the plane stress approximation: a Mindlin-Reissner like plate model and the more general model (1.1) which will be called in the following the solid plate model, since it is the restriction to a plane geometry of a solid shell model (see [25] for instance) as long as the finite element approximation is not concerned.

The rest of the paper is outlined as follows. In Section 2 we introduce the classical formulation of the 3D frictional contact problem, with some specific notations from plate theory. We focus on a model problem which consists of a unilateral contact between an elastic body and a rigid support (Signorini's problem). Then, we introduce Nitsche's method for the contact condition. Section 3 is devoted to obtaining the Nitsche-based formulation for the classical Kirchhoff-Love and Mindlin-Reissner plate models. We obtain in Section 4 the Nitsche-based formulation for two additional solid plate models: the first one is based on Mindlin-Reissner kinematics but without the plane-stress assumption and the second one takes into account the enriched kinematics (1.1). In Section 5, the different approach of Nitsche's method proposed in [24] for the Kirchhoff-Love plate model is recalled for comparison. Then, Section 6 deals with the finite element approximation of the proposed formulations and presents some numerical tests. The convergence in $L^{2}$-norm and $H^{1}$-semi-norm of the displacement toward a fully 3D-solution computed on a very fine mesh is analyzed. A brief study of the choice of Nitsche's parameter is given in Section 7 and the paper is ended by some concluding remarks in Section 8 .

\section{3D-CONTACT PROBLEMS: WEAK FORMULATIONS}

\subsection{Loading and boundary conditions}

The boundary $\partial \Omega^{\varepsilon}$ of the domain $\Omega^{\varepsilon}$ is assumed to be partitioned into three parts. Due to the particular shape of the domain, the partition is composed of the "lateral" one $\Gamma_{0}^{\varepsilon}$, the "upper" one $\Gamma_{+}^{\varepsilon}$ and the "lower" one $\Gamma_{-}^{\varepsilon}$, i.e.

$$
\partial \Omega^{\varepsilon}=\Gamma_{0}^{\varepsilon} \cup \Gamma_{+}^{\varepsilon} \cup \Gamma_{-}^{\varepsilon}, \quad \Gamma_{0}^{\varepsilon}=\partial \Omega \times(-\varepsilon, \varepsilon), \quad \Gamma_{+}^{\varepsilon}=\Omega \times\{\varepsilon\}, \quad \Gamma_{-}^{\varepsilon}=\Omega \times\{-\varepsilon\} .
$$

We suppose that the lateral part $\Gamma_{0}^{\varepsilon}$ consists in two non-overlapping sub-parts $\Gamma_{D}^{\varepsilon}$ and $\Gamma_{N}^{\varepsilon}$. On $\Gamma_{D}^{\varepsilon}\left(\operatorname{resp} . \Gamma_{N}^{\varepsilon}\right)$ displacements $u$ (resp. tractions) are prescribed. We consider the following situation:

- For the sake of simplicity, the body is clamped on $\Gamma_{D}^{\varepsilon}:=\Gamma_{D} \times(-\varepsilon, \varepsilon)$ which is assumed to be a non-zero Lebesgue measure part of the boundary $\Gamma_{0}^{\varepsilon} \subset \partial \Omega^{\varepsilon}$, that is to say $u^{\varepsilon}=0$ on $\Gamma_{D}^{\varepsilon}$, and is traction free on the complementary part $\Gamma_{N}^{\varepsilon}$. 


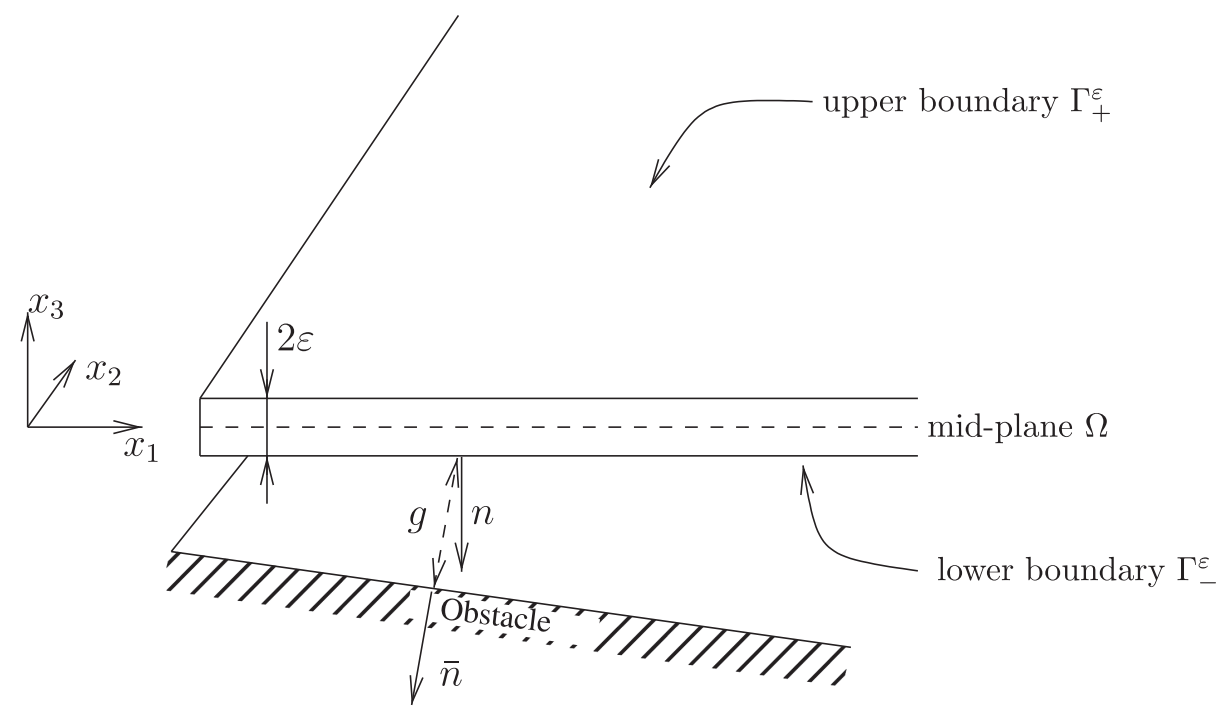

FIGURE 1. Plate in unilateral contact with a rigid obstacle.

- In addition, the body can be subjected to a volume force $f^{V} \in L^{2}\left(\Omega^{\varepsilon} ; \mathbb{R}^{3}\right)$ (such as gravity).

- As far as loading is concerned, the upper part, $\Gamma_{+}^{\varepsilon}$, is loaded by a surface force $\ell \in L^{2}\left(\Gamma_{+}^{\varepsilon} ; \mathbb{R}^{3}\right)$.

- The actual surface on which the body comes into contact with the obstacle is not known in advance, but is assumed contained in $\Gamma_{-}^{\varepsilon}$.

The space of admissible displacements for the three-dimensional problem is

$$
\mathbb{V}^{\varepsilon}:=\left\{v \in H^{1}\left(\Omega^{\varepsilon} ; \mathbb{R}^{3}\right) \mid v=0 \quad \text { on } \quad \Gamma_{D}^{\varepsilon}\right\} .
$$

\subsection{Unilateral boundary conditions and weak form}

On the potential contact boundary $\Gamma_{-}^{\varepsilon}$, the vector $n$ still denote the outward unit vector $\left(n=-e_{3}\right.$, here). Moreover, we also consider the unit vector $\bar{n}$, the contact normal. In common models, $\bar{n}$ can be chosen different from $n$ (see Fig. 1), for instance, to be the normal vector to the obstacle or, in a two-body contact, the normal vector to a second deformable body or even to an intermediate surface.

We decompose the displacement field $u: \Omega^{\varepsilon} \longrightarrow \mathbb{R}^{3}$ and density of surface forces $\sigma(u) n: \Gamma_{-}^{\varepsilon} \longrightarrow \mathbb{R}$ in normal and tangential parts as follows:

$$
u=u_{n} \bar{n}+u_{t} \quad \text { and } \quad \sigma(u) n=\sigma_{n}(u) \bar{n}+\sigma_{t}(u) .
$$

Let us now introduce the static equation with Signorini's conditions along the plate. We assume that the motion of the plate is limited by a rigid obstacle, located below the plate, determined by

$$
g: \Omega^{\varepsilon} \longrightarrow \mathbb{R} \cup\{+\infty\},
$$

such that the displacement is constrained to belong to the convex set $\mathbb{K}^{\varepsilon} \subset \mathbb{V}^{\varepsilon}$ given by

$$
\mathbb{K}^{\varepsilon}:=\left\{v \in \mathbb{V}^{\varepsilon} \mid v_{n} \leq g \quad \text { on } \quad \Omega^{\varepsilon}\right\} .
$$

The three-dimensional contact problem in linear elasticity consists in finding the displacement field $u$ and the traction $\sigma(u) n$ verifying the strong equation (2.1) and the contact conditions described hereafter: 


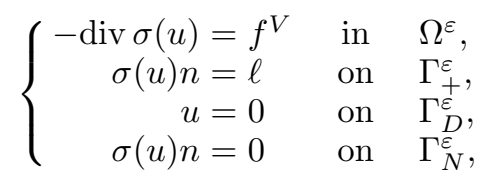

where $\sigma$ stands for the stress tensor field and div denotes the divergence operator of tensor valued functions. Denoting $\gamma$ the linearized strain tensor field

$$
\gamma_{i j}(u):=\frac{1}{2}\left(\partial_{i} u_{j}+\partial_{j} u_{i}\right)
$$

we consider Hooke's law for isotropic material:

$$
\sigma_{i j}=\frac{E \nu}{(1+\nu)(1-2 \nu)} \gamma_{k k} \delta_{i j}+\frac{E}{2(1+\nu)} \gamma_{i j}
$$

The unilateral contact conditions (or Kuhn-Tucker conditions) are given by:

$$
u_{n} \leq g, \quad \sigma_{n}(u) \leq 0 \quad \text { and } \quad\left(u_{n}-g\right) \sigma_{n}(u)=0 \quad \text { on } \quad \Gamma_{-}^{\varepsilon} .
$$

In the frictionless contact case, this condition is simply completed by

$$
\sigma_{t}(u)=0, \quad \text { on } \quad \Gamma_{-}^{\varepsilon},
$$

whereas the so-called Coulomb's static friction condition on $\Gamma_{-}^{\varepsilon}$ reads

$$
\begin{cases}\left\|\sigma_{t}(u)\right\| \leq-\mathcal{F} \sigma_{n}(u) & \text { if } \quad u_{t}=0 \\ \sigma_{t}(u)=\mathcal{F} \sigma_{n}(u) \frac{u_{t}}{\left\|u_{t}\right\|} & \text { otherwise }\end{cases}
$$

where $\mathcal{F} \geq 0$ is the Coulomb coefficient of friction and $\|\cdot\|$ stands for the euclidean norm in $\mathbb{R}^{3}$.

Remark 2.1. Recall that the Coulomb's static friction condition is rather artificial but that is leads to an interesting intermediate problem. The true Coulomb's friction law involves tangential contact velocity and not tangential displacement. However, a problem similar to the one discussed here is obtained by time discretization of the quasi-static frictional contact evolution problem. In this case $u, f$ and $\ell$ stand for $u((i+1) \Delta t), f((i+1) \Delta t))$ and $\ell((i+1) \Delta t)$ respectively and $u_{t}$ has to be replaced by $\left[u_{t}((i+1) \Delta t)-u_{t}(i \Delta t)\right] / \Delta t$, where $\Delta t$ denotes the time step. For simplicity and without any loss of generality only the static case described above will be considered in the following.

From Green's formula and equation (2.1), we get the weak variational formulation for all $v \in \mathbb{V}^{\varepsilon}$ :

$$
\int_{\Omega^{\varepsilon}} \sigma(u): \gamma(v) \mathrm{d} \Omega-\int_{\Gamma_{-}^{\varepsilon}} \sigma(u) n \cdot v \mathrm{~d} \Gamma=\int_{\Omega^{\varepsilon}} f^{V} \cdot v \mathrm{~d} \Omega+\int_{\Gamma_{+}^{\varepsilon}} \ell \cdot v \mathrm{~d} \Gamma .
$$

In the following, the membrane stress resultants $\left(N_{\alpha \beta}\right)$, the bending moments stress resultants $\left(M_{\alpha \beta}\right), Q_{\beta}, P$ the pinch stress and the shear stress $\left(T_{\beta}\right)$ are defined as (for more details see $[15,17]$ )

$$
\begin{aligned}
N_{\alpha \beta} & :=\int_{-\varepsilon}^{\varepsilon} \sigma_{\alpha \beta}(u) \mathrm{d} x_{3}, \\
M_{\alpha \beta} & :=\int_{-\varepsilon}^{\varepsilon} x_{3} \sigma_{\alpha \beta}(u) \mathrm{d} x_{3}, \\
T_{\beta} & :=\int_{-\varepsilon}^{\varepsilon} \sigma_{\beta 3}(u) \mathrm{d} x_{3}, \\
Q_{\beta} & :=\int_{-\varepsilon}^{\varepsilon} x_{3} \sigma_{\beta 3}(u) \mathrm{d} x_{3}, \\
P & :=\int_{-\varepsilon}^{\varepsilon} \sigma_{33}(u) \mathrm{d} x_{3} .
\end{aligned}
$$


Remark 2.2. The Korn inequality that is satisfied for this problem is simply adapted from the classical one, which allows to prove the existence and the uniqueness of the solution to problem (2.5) subject to (2.2) and (2.3) for any fixed $\varepsilon>0$, thanks to Lax-Milgram theorem, see [21]. For the general problem (2.5) subject to (2.2) and (2.4) the existence of a solution is only available for small Coulomb coefficient of friction $\mathcal{F} \geq 0$ (see $[19,26,28])$.

\subsection{Weak formulation using Nitsche's method}

A reformulation of the previous contact conditions comes from the augmented Lagrangian formulation of contact problems. Let $r$ be a given real positive number. As in $[1,9,10]$, the contact conditions $(2.2)$ are rewritten as

$$
\sigma_{n}(u)=-\left[\sigma_{n}(u)+r\left(g-u_{n}\right)\right]_{-},
$$

where we denote $[x]_{-}:=(|x|-x) / 2$ the negative part. We can reformulate as well Coulomb's friction condition using the projection $P_{B(0, s)}$ onto the ball $B(0, s)$ defined by

$$
\mathbf{P}_{B(0, s)}(q):= \begin{cases}\Pi_{t}(q) & \text { if }\left\|\Pi_{t}(q)\right\| \leq s \\ s \frac{\Pi_{t}(q)}{\left\|\Pi_{t}(q)\right\|} & \text { otherwise }\end{cases}
$$

where $\Pi_{t}$ is the tangential projection defined as $\Pi_{t}:=\mathbf{I}-n \otimes n$, with $\mathbf{I}$ the identity operator. Still for a given real positive number $r$, Coulomb's friction condition is equivalent to the non-smooth equation

$$
\sigma_{t}(u)=\mathbf{P}_{B\left(0, \mathcal{F}\left[\sigma_{n}(u)+r\left(g-u_{n}\right)\right]_{-}\right)}\left(\sigma_{t}(u)-r u_{t}\right) .
$$

Let now $\mathcal{O} \in \mathbb{R}$ be a fixed parameter that we use to recover different variants of the Nitsche's method, as in the 3D linear elastic setting (see, e.g. [10]) for $\mathcal{O}=1,0,-1$ corresponding to symmetric, non-symmetric and skew-symmetric contributions of the contact terms, respectively. We insert finally the expressions (2.6) and (2.7) into (2.5) and obtain, formally, our Nitsche-based formulation for frictional contact. In the decomposition

$$
\int_{\Gamma_{-}^{\varepsilon}} \sigma(u) n \cdot v \mathrm{~d} \Gamma=\int_{\Gamma_{-}^{\varepsilon}} \sigma_{n}(u) v_{n}(v) \mathrm{d} \Gamma+\int_{\Gamma_{-}^{\varepsilon}} \sigma_{t}(u) \cdot v_{t} \mathrm{~d} \Gamma
$$

the normal contact term (obtained from (2.6) and the classical decomposition introduced in $[10,11,20]$ ) can be written:

$$
\begin{aligned}
\int_{\Gamma_{-}^{\varepsilon}} \sigma_{n}(u) v_{n} \mathrm{~d} \Gamma & =\frac{1}{r} \int_{\Gamma^{\varepsilon}} \sigma_{n}(u)\left(r v_{n}-\mathcal{O} \sigma_{n}(v)\right) \mathrm{d} \Gamma+\frac{\mathcal{O}}{r} \int_{\Gamma_{-}^{\varepsilon}} \sigma_{n}(u) \sigma_{n}(v) \mathrm{d} \Gamma \\
& =-\frac{1}{r} \int_{\Gamma_{-}^{\varepsilon}}\left[\sigma_{n}(u)+r\left(g-u_{n}\right)\right]_{-}\left(r v_{n}-\mathcal{O} \sigma_{n}(v)\right) \mathrm{d} \Gamma+\frac{\mathcal{O}}{r} \int_{\Gamma_{-}^{\varepsilon}} \sigma_{n}(u) \sigma_{n}(v) \mathrm{d} \Gamma \\
& =-\left.\frac{1}{r} \int_{\Omega}\left[\sigma_{n}(u)+r\left(g-u_{n}\right)\right]_{-}\left(r v_{n}-\mathcal{O} \sigma_{n}(v)\right)\right|_{x_{3}=-\varepsilon} \mathrm{d} \Omega+\left.\frac{\mathcal{O}}{r} \int_{\Omega} \sigma_{n}(u) \sigma_{n}(v)\right|_{x_{3}=-\varepsilon} \mathrm{d} \Omega
\end{aligned}
$$

and with frictional contact term:

$$
\begin{aligned}
\int_{\Gamma_{-}^{\varepsilon}} \sigma_{t}(u) \cdot v_{t} \mathrm{~d} \Gamma= & \left.\frac{1}{r} \int_{\Omega} \mathbf{P}_{B\left(0,-\mathcal{F}\left[\sigma_{n}(u)+r\left(g-u_{n}\right)\right]_{-}\right)}\left(\sigma_{t}(u)-r u_{t}\right) \cdot\left(r v_{t}-\mathcal{O} \sigma_{t}(v)\right)\right|_{x_{3}=-\varepsilon} \mathrm{d} \Omega \\
& +\left.\frac{\mathcal{O}}{r} \int_{\Omega} \sigma_{t}(u) \cdot \sigma_{t}(v)\right|_{x_{3}=-\varepsilon} \mathrm{d} \Omega .
\end{aligned}
$$


Now, inserting the expressions (2.9) and (2.10) of (2.8) into the weak problem (2.5), we obtain the so-called Nitsche-based method:

$$
\left\{\begin{array}{c}
\int_{\Omega^{\varepsilon}} \sigma(u): \gamma(v) \mathrm{d} \Omega+\left.\frac{1}{r} \int_{\Omega}\left[\sigma_{n}(u)+r\left(g-u_{n}\right)\right]_{-}\left(r v_{n}-\mathcal{O} \sigma_{n}(v)\right)\right|_{x_{3}=-\varepsilon} \mathrm{d} \Omega \\
-\left.\frac{1}{r} \int_{\Omega} \mathbf{P}_{B\left(0, \mathcal{F}\left[\sigma_{n}(u)+r\left(g-u_{n}\right)\right]_{-}\right)}\left(\sigma_{t}(u)-r u_{t}\right) \cdot\left(r v_{t}-\mathcal{O} \sigma_{t}(v)\right)\right|_{x_{3}=-\varepsilon} \mathrm{d} \Omega \\
-\left.\frac{\mathcal{O}}{r} \int_{\Omega}(\sigma(u) n) \cdot(\sigma(v) n)\right|_{x_{3}=-\varepsilon} \mathrm{d} \Omega=\int_{\Omega^{\varepsilon}} f^{V} \cdot v \mathrm{~d} \Omega+\int_{\Gamma_{+}^{\varepsilon}} \ell \cdot v \mathrm{~d} \Gamma
\end{array}\right.
$$

Note that because of the terms in $\sigma(u) n$ and $\sigma(v) n$, this formulation only makes sense for both $\sigma(u) n$ and $\sigma(v) n$ in $L^{2}\left(\gamma_{-}^{\varepsilon} ; \mathbb{R}^{3}\right)$ (except for $\mathcal{F}=\mathcal{O}=0$ ). In our case, this regularity is automatically obtained for $u, v \in \mathbb{V}^{\varepsilon}$ satisfying additionally the kinematic assumptions (1.1) which are considered below. Note that with these assumptions, the decomposition into normal and tangential components is as follows:

$$
\begin{aligned}
v_{t} & :=v-v_{n} \bar{n}=v-\left(\underline{v}_{\alpha} \bar{n}_{\alpha}+x_{3} \psi_{\alpha} \bar{n}_{\alpha}+v_{3} \bar{n}_{3}+x_{3} \psi_{3} \bar{n}_{3}\right) \bar{n}, \\
u_{t} & :=u-u_{n} \bar{n}=u-\left(\underline{u}_{\alpha} \bar{n}_{\alpha}+x_{3} \theta_{\alpha} \bar{n}_{\alpha}+u_{3} \bar{n}_{3}+x_{3} \theta_{3} \bar{n}_{3}\right) \bar{n} .
\end{aligned}
$$

\section{WEAK FORMULATiOn FOR CLASSiCAL PLATE MODELS UNDER PLANE STRESS ASSUMPTION}

\subsection{Weak formulation under Kirchhoff-Love assumptions}

We now aim to make explicit each term of the formulation (2.11) under Kirchhoff-Love assumptions. The plane stress assumption consists in assuming that the components $\sigma_{i 3}(u)$ are negligible compared to $\sigma_{\alpha \beta}(u)$ which, for an isotropic material under Hooke's law, leads to the relations

$$
\gamma_{\alpha \beta}(u)=\frac{1+\nu}{E} \sigma_{\alpha \beta}(u)-\frac{\nu}{E} \sigma_{\eta \eta}(u) \delta_{\alpha \beta}, \quad \sigma_{\alpha \beta}(u)=\frac{E}{1-\nu^{2}}\left[(1-\nu) \gamma_{\alpha \beta}(u)+\nu \gamma_{\eta \eta}(u) \delta_{\alpha \beta}\right] .
$$

The linearized strain tensor field reads in that case

$$
\gamma_{\alpha \beta}(u)=\gamma_{\alpha \beta}(\underline{u})-x_{3} \partial_{\alpha \beta}^{2} u_{3}
$$

and the stress tensor

$$
\sigma_{\alpha \beta}(u)=\frac{E}{1-\nu^{2}}\left((1-\nu)\left[\gamma_{\alpha \beta}(\underline{u})-x_{3} \partial_{\alpha \beta}^{2} u_{3}\right]+\nu\left[\gamma_{\eta \eta}(\underline{u})-x_{3} \partial_{\eta \eta}^{2} u_{3}\right] \delta_{\alpha \beta}\right) .
$$

Then, we obtain

$$
\int_{\Omega^{\varepsilon}} \sigma(u): \gamma(v) \mathrm{d} \Omega=\int_{\Omega}[\underbrace{\left(\int_{-\varepsilon}^{\varepsilon} \sigma_{\alpha \beta}(u) \mathrm{d} x_{3}\right)}_{N_{\alpha \beta}} \gamma_{\alpha \beta}(\underline{v})-\underbrace{\left(\int_{-\varepsilon}^{\varepsilon} x_{3} \sigma_{\alpha \beta}(u) \mathrm{d} x_{3}\right)}_{M_{\alpha \beta}} \partial_{\alpha \beta}^{2} v_{3}] \mathrm{d} \Omega .
$$

Thanks to the model and according to Hooke's law, the membrane stress resultants $\left(N_{\alpha \beta}\right)$ and the bending moments stress resultants $\left(M_{\alpha \beta}\right)$ read

$$
\begin{aligned}
N_{\alpha \beta} & =\int_{-\varepsilon}^{\varepsilon} \sigma_{\alpha \beta}(u) \mathrm{d} x_{3}=\frac{2 \varepsilon E}{1-\nu^{2}}\left[(1-\nu) \gamma_{\alpha \beta}(\underline{u})+\nu \gamma_{\eta \eta}(\underline{u}) \delta_{\alpha \beta}\right], \\
M_{\alpha \beta} & =\int_{-\varepsilon}^{\varepsilon} x_{3} \sigma_{\alpha \beta}(u) \mathrm{d} x_{3}=-\frac{2 \varepsilon^{3} E}{3\left(1-\nu^{2}\right)}\left[(1-\nu) \partial_{\alpha \beta}^{2} u_{3}+\nu \partial_{\eta \eta}^{2} u_{3} \delta_{\alpha \beta}\right] .
\end{aligned}
$$


We rewrite the right-hand side of (2.11) as follows:

$$
\int_{\Omega^{\varepsilon}} f^{V} \cdot v \mathrm{~d} \Omega+\int_{\Gamma_{+}^{\varepsilon}} \ell \cdot v \mathrm{~d} \Gamma=\int_{\Omega} F_{i} v_{i}-G_{\alpha} \partial_{\alpha} v_{3} \mathrm{~d} \Omega
$$

with

$$
F_{i}:=\int_{-\varepsilon}^{\varepsilon} f_{i}^{V} \mathrm{~d} x_{3}+\ell_{i}, \quad G_{\alpha}:=\int_{-\varepsilon}^{\varepsilon} x_{3} f_{\alpha}^{V} \mathrm{~d} x_{3}+\varepsilon \ell_{\alpha} .
$$

Neglecting the components $\sigma_{i 3}(u)$ according to the plane stress assumption, the terms involved in (2.9) can be expressed as follows:

$$
\begin{aligned}
{\left[\sigma_{n}(u)+r\left(g-u_{n}\right)\right]_{-} } & =\left[\sigma_{\alpha \beta}(u) n_{\beta} \bar{n}_{\alpha}+r\left(g-\underline{u}_{\alpha} \bar{n}_{\alpha}-\varepsilon \partial_{\alpha} u_{3} \bar{n}_{\alpha}-u_{3} \bar{n}_{3}\right)\right]_{-} \\
r v_{n}-\mathcal{O} \sigma_{n}(v) & =r\left(\underline{v}_{\alpha} \bar{n}_{\alpha}+\varepsilon \partial_{\alpha} v_{3} \bar{n}_{\alpha}+v_{3} \bar{n}_{3}\right)-\mathcal{O}\left(\sigma_{\alpha \beta}(v) n_{\beta} \bar{n}_{\alpha}\right) .
\end{aligned}
$$

Now, the term in the frictional part (2.10) can be made explicit as

$$
\sigma_{t}(u)-r u_{t}=\sigma_{\alpha \beta}(u) n_{\beta} e_{\alpha}-\left(\sigma_{\alpha \beta}(u) n_{\beta} \bar{n}_{\alpha}\right) \bar{n}-r\left(u-u_{n} \bar{n}\right) .
$$

In our situation, we have $n=-e_{3}$, then the three last expressions are then simplified into:

$$
\begin{aligned}
{\left[\sigma_{n}(u)+r\left(g-u_{n}\right)\right]_{-} } & =r\left[g-\underline{u}_{\alpha} \bar{n}_{\alpha}-\varepsilon \partial_{\alpha} u_{3} \bar{n}_{\alpha}-u_{3} \bar{n}_{3}\right]_{-}, \\
r v_{n}-\mathcal{O} \sigma_{n}(v) & =r\left(\underline{v}_{\alpha} \bar{n}_{\alpha}+\varepsilon \partial_{\alpha} v_{3} \bar{n}_{\alpha}+v_{3} \bar{n}_{3}\right), \\
\sigma_{t}(u)-r u_{t} & =-r u_{t} .
\end{aligned}
$$

\section{Special case $\bar{n}=n=-e_{3}$}

In the special, but common case $\bar{n}=n=-e_{3}$, the terms (3.1)-(3.3) reduce to:

$$
\begin{aligned}
{\left[\sigma_{n}(u)+r\left(g-u_{n}\right)\right]_{-} } & =r\left[g+\underline{u}_{3}\right]_{-}, \\
r v_{n}-\mathcal{O} \sigma_{n}(v) & =-r \underline{v}_{3}, \\
\sigma_{t}(u)-r u_{t} & =-r u_{t}=-r\left(\underline{u}_{\alpha} e_{\alpha}-\varepsilon \partial_{\alpha} \underline{u}_{3} e_{\alpha}\right) .
\end{aligned}
$$

Finally, denoting

$$
\mathbb{V}_{\mathrm{KL}}:=\left\{\underline{v} \in H^{1}\left(\Omega ; \mathbb{R}^{3}\right) \mid \underline{v}_{3} \in H^{2}(\Omega), \quad \underline{v}=0 \quad \text { on } \quad \Gamma_{D}, \quad \partial_{n} \underline{v}_{3}=0 \quad \text { on } \quad \Gamma_{D}\right\},
$$

we end up with the following formulation considering the Kirchhoff-Love assumptions, still in the case $\bar{n}=n=$ $-e_{3}$ :

$$
\left\{\begin{array}{l}
\text { Find } \underline{u} \in \mathbb{V}_{\mathrm{KL}} \text { such that for all } \underline{v} \in \mathbb{V}_{\mathrm{KL}} \\
\int_{\Omega} N_{\alpha \beta} \gamma_{\alpha \beta}(\underline{v})-M_{\alpha \beta} \partial_{\alpha \beta}^{2} \underline{v}_{3} \mathrm{~d} \Omega-r \int_{\Omega}\left[g+\underline{u}_{3}\right]_{-} \underline{v}_{3} \mathrm{~d} \Omega \\
\quad+r \int_{\Omega} \mathbf{P}_{B\left(0, r \mathcal{F}\left[g+\underline{u}_{3}\right]_{-}\right)}\left(\underline{u}_{\alpha} e_{\alpha}-\varepsilon \partial_{\alpha} \underline{u}_{3} e_{\alpha}\right) \cdot\left(\underline{v}_{\alpha} e_{\alpha}-\varepsilon \partial_{\alpha} \underline{v}_{3} e_{\alpha}\right) \mathrm{d} \Omega \\
\quad=\int_{\Omega} F_{i} \underline{v}_{i}-G_{\alpha} \partial_{\alpha} \underline{v}_{3} \mathrm{~d} \Omega .
\end{array}\right.
$$

We can now briefly review the remaining terms when Kirchhoff-Love assumptions are taken into account. The terms involved in unilateral contact and friction conditions are reduced to $\sigma_{n}(u)+r\left(g-u_{n}\right)=r\left(g+u_{3}\right)$ and $\sigma_{t}(u)-r u_{t}=-r u_{t}$ (in the case $\bar{n}=n=-e_{3}$ ), which means that both contact and friction are approximated by a penalty method with $r$ as the penalty coefficient. Indeed, with Kirchhoff-Love assumptions, no additional contribution than penalty terms can be obtained with the proposed Nitsche's method mainly because of the plane stress approximation. 


\subsection{Weak formulation under Mindlin-Reissner assumptions}

Let us now perform the same analysis but considering Mindlin-Reissner assumptions. In the Mindlin-Reissner plate model, the pinch stress $\sigma_{33}$ is still neglected and Hooke's law still leads to the relations

$$
\gamma_{\alpha \beta}(u)=\frac{1+\nu}{E} \sigma_{\alpha \beta}(u)-\frac{\nu}{E} \sigma_{\eta \eta}(u) \delta_{\alpha \beta}, \quad \sigma_{\alpha \beta}(u)=\frac{E}{1-\nu^{2}}\left[(1-\nu) \gamma_{\alpha \beta}(u)+\nu \gamma_{\eta \eta}(u) \delta_{\alpha \beta}\right] .
$$

However, the shear stress is no longer neglected and is approximated by

$$
\sigma_{\alpha 3}(u)=\kappa \frac{E}{1+\nu} \gamma_{\alpha 3}(u)
$$

with $\kappa$ the shear correction factor [5], which standard value is $\kappa=\frac{5}{6}$. The linearized strain tensor field reads in that case

$$
\gamma_{\alpha \beta}(u)=\frac{1}{2}\left(\partial_{\alpha} u_{\beta}+\partial_{\beta} u_{\alpha}\right)=\gamma_{\alpha \beta}(\underline{u})+x_{3} \gamma_{\alpha \beta}(\theta) \quad \text { and } \quad \gamma_{\alpha 3}(u)=\frac{1}{2}\left(\partial_{\alpha} u_{3}+\theta_{\alpha}\right),
$$

and with

$$
\begin{aligned}
\sigma_{\alpha \beta}(u) & =\frac{E}{1-\nu^{2}}\left((1-\nu)\left[\gamma_{\alpha \beta}(\underline{u})-x_{3} \gamma_{\alpha \beta}(\theta)\right]+\nu\left[\gamma_{\eta \eta}(\underline{u})-x_{3} \gamma_{\eta \eta}(\theta)\right] \delta_{\alpha \beta}\right), \\
\sigma_{\alpha 3}(u) & =\kappa \frac{E}{2(1+\nu)}\left(\partial_{\alpha} u_{3}+\theta_{\alpha}\right) .
\end{aligned}
$$

The work of the internal efforts end up taking the form

$$
\begin{aligned}
\int_{\Omega^{\varepsilon}} \sigma(u): \gamma(v) \mathrm{d} \Omega= & \int_{\Omega}[\underbrace{\left(\int_{-\varepsilon}^{\varepsilon} \sigma_{\alpha \beta}(u) \mathrm{d} x_{3}\right)}_{N_{\alpha \beta}} \gamma_{\alpha \beta}(\underline{v})+\underbrace{\left(\int_{-\varepsilon}^{\varepsilon} x_{3} \sigma_{\alpha \beta}(u) \mathrm{d} x_{3}\right)}_{M_{\alpha \beta}} \gamma_{\alpha \beta}(\psi)] \mathrm{d} \Omega \\
& +\int_{\Omega}[\underbrace{\left(\int_{-\varepsilon}^{\varepsilon} \sigma_{\beta 3} \mathrm{~d} x_{3}\right)}_{T_{\beta}}\left(\partial_{\beta} v_{3}+\psi_{\beta}\right)] \mathrm{d} \Omega .
\end{aligned}
$$

Thanks to the model and according to Hooke's law, the membrane stress resultants $\left(N_{\alpha \beta}\right)$, the bending moments stress resultants $\left(M_{\alpha \beta}\right)$ and the shear stress resultants $\left(T_{\beta}\right)$ are given by

$$
\begin{aligned}
N_{\alpha \beta} & =\frac{2 \varepsilon E}{1-\nu^{2}}\left[(1-\nu) \gamma_{\alpha \beta}(\underline{u})+\nu \gamma_{\eta \eta}(\underline{u}) \delta_{\alpha \beta}\right], \\
M_{\alpha \beta} & =\frac{2 \varepsilon^{3} E}{3\left(1-\nu^{2}\right)}\left[(1-\nu) \gamma_{\alpha \beta}(\theta)+\nu \gamma_{\eta \eta}(\theta) \delta_{\alpha \beta}\right], \\
T_{\beta} & =\frac{\varepsilon \kappa E}{1+\nu}\left[\partial_{\beta} u_{3}+\theta_{\beta}\right] .
\end{aligned}
$$

The right-hand side of (2.11) is now rewritten as

$$
\int_{\Omega^{\varepsilon}} f^{V} \cdot v \mathrm{~d} \Omega+\int_{\Gamma_{+}^{\varepsilon}} \ell \cdot v \mathrm{~d} \Gamma=\int_{\Omega} F_{i} v_{i}-G_{\alpha} \psi_{\alpha} \mathrm{d} \Omega
$$

with still

$$
F_{i}=\int_{-\varepsilon}^{\varepsilon} f_{i}^{V} \mathrm{~d} x_{3}+\ell_{i}, \quad G_{\alpha}=\int_{-\varepsilon}^{\varepsilon} x_{3} f_{\alpha}^{V} \mathrm{~d} x_{3}+\varepsilon \ell_{\alpha} .
$$


We pass to the non-penetration condition terms from (2.9). We obtain

$$
\begin{aligned}
{\left[\sigma_{n}(u)+r\left(g-u_{n}\right)\right]_{-} } & =\left[\sigma_{i j}(u) n_{j} \bar{n}_{i}+r\left(g-\underline{u}_{\alpha} \bar{n}_{\alpha}-\varepsilon \theta_{\alpha} \bar{n}_{\alpha}-u_{3} \bar{n}_{3}\right)\right]_{-} \\
r v_{n}-\mathcal{O} \sigma_{n}(v) & =r\left(\underline{v}_{\alpha} \bar{n}_{\alpha}+\varepsilon \psi_{\alpha} \bar{n}_{\alpha}+v_{3} \bar{n}_{3}\right)-\mathcal{O} \sigma_{i j}(v) n_{j} \bar{n}_{i},
\end{aligned}
$$

still assuming $\sigma_{33}=0$ and the corresponding expression for $\sigma_{\alpha \beta}(v), \sigma_{33}(v)$ and $\sigma_{\alpha 3}(v)$. The term in the friction condition (2.10) reads now

$$
\sigma_{t}(u)-r u_{t}=\sigma_{i j}(u) n_{j} e_{i}-\left(\sigma_{i j}(u) n_{j} \bar{n}_{i}\right) \bar{n}-r\left(u-u_{n} \bar{n}\right),
$$

and the simplifications coming from $n=-e_{3}$ leads to

$$
\begin{aligned}
{\left[\sigma_{n}(u)+r\left(g-u_{n}\right)\right]_{-} } & =\left[-\sigma_{\alpha 3}(u) \bar{n}_{\alpha}+r\left(g-\underline{u}_{\alpha} \bar{n}_{\alpha}-\varepsilon \theta_{\alpha} \bar{n}_{\alpha}-u_{3} \bar{n}_{3}\right)\right]_{-}, \\
r v_{n}-\mathcal{O} \sigma_{n}(v) & =r\left(\underline{v}_{\alpha} \bar{n}_{\alpha}+\varepsilon \psi_{\alpha} \bar{n}_{\alpha}+v_{3} \bar{n}_{3}\right)+\mathcal{O} \sigma_{\alpha 3}(v) \bar{n}_{\alpha}, \\
\sigma_{t}(u)-r u_{t} & =\sigma_{\alpha 3}(u)\left(\bar{n}_{\alpha} \bar{n}-e_{\alpha}\right)-r u_{t} .
\end{aligned}
$$

\section{Special case $\bar{n}=n=-e_{3}$}

In the special case $\bar{n}=n=-e_{3}$, we obtain:

$$
\begin{aligned}
{\left[\sigma_{n}(u)+r\left(g-u_{n}\right)\right]_{-} } & =r\left[\left(g+\underline{u}_{3}\right)\right]_{-}, \\
r v_{n}-\mathcal{O} \sigma_{n}(v) & =-r \underline{v}_{3}, \\
\sigma_{t}(u)-r u_{t} & =-\sigma_{\alpha 3}(u) e_{\alpha}-r u_{t}=-\frac{\kappa E}{2(1+\nu)}\left(\partial_{\alpha} \underline{u}_{3}+\theta_{\alpha}\right) e_{\alpha}-r\left(\underline{u}_{\alpha} e_{\alpha}+\varepsilon \theta_{\alpha} e_{\alpha}\right), \\
r v_{t}-\mathcal{O} \sigma_{t}(v) & =r v_{t}+\mathcal{O} \sigma_{\alpha 3}(v) e_{\alpha}=r\left(\underline{v}_{\alpha} e_{\alpha}+\varepsilon \psi_{\alpha} e_{\alpha}\right)+\mathcal{O} \frac{\kappa E}{2(1+\nu)}\left(\partial_{\alpha} \underline{v}_{3}+\psi_{\alpha}\right) e_{\alpha} .
\end{aligned}
$$

Finally, denoting

$$
\mathbb{V}_{\mathrm{MR}}:=\left\{(\underline{v}, \psi) \in H^{1}\left(\Omega ; \mathbb{R}^{3}\right) \times H^{1}\left(\Omega ; \mathbb{R}^{2}\right) \mid \underline{v}=0 \quad \text { on } \quad \Gamma_{D}, \quad \psi=0 \quad \text { on } \quad \Gamma_{D}\right\},
$$

we end up with the following formulation under the Mindlin-Reissner assumptions, in the case $\bar{n}=n=-e_{3}$ :

$$
\left\{\begin{array}{l}
\text { Find }(\underline{u}, \theta) \in \mathbb{V}_{\mathrm{MR}} \text { such that for all }(\underline{v}, \psi) \in \mathbb{V}_{\mathrm{MR}} \\
\int_{\Omega} N_{\alpha \beta} \gamma_{\alpha \beta}(\underline{v})+M_{\alpha \beta} \gamma_{\alpha \beta}(\psi)+T_{\beta}\left(\partial_{\beta} \underline{v}_{3}+\psi_{\beta}\right) \mathrm{d} \Omega-r \int_{\Omega}\left[g+\underline{u}_{3}\right]_{-} \underline{v}_{3} \mathrm{~d} \Omega \\
\quad+\frac{1}{r} \int_{\Omega} \mathbf{P}_{B\left(0, r \mathcal{F}\left[g+\underline{u}_{3}\right]_{-}\right)}\left(\sigma_{\alpha 3}(u) e_{\alpha}+r u_{t}\right) \cdot\left(r v_{t}+\mathcal{O} \sigma_{\alpha 3}(v) e_{\alpha}\right) \mathrm{d} \Omega \\
\quad-\frac{\mathcal{O}}{r} \int_{\Omega} \sigma_{\alpha 3}(u) \sigma_{\alpha 3}(v) \mathrm{d} \Omega=\int_{\Omega} F_{i} v_{i}-G_{\alpha} \psi_{\alpha} \mathrm{d} \Omega .
\end{array}\right.
$$

Note in particular the presence of the shear stress in the friction term $\sigma_{t}(u)-r u_{t}$ which disappear in the Kirchhoff-Love plate model. This means that the friction condition is no longer reduced to a penalty approximation. The Mindlin-Reissner model is richer in this sense. However, the contact term remains a pure penalty term. 


\section{WEAK FORMULATION FOR SOME SIMPLE SOLID PLATE MODELS}

\subsection{Weak formulation under Mindlin-Reissner kinematics without any plane stress approximation}

To retrieve a non-trivial Nitsche contact term, it is necessary to abandon the plane stress approximation $\sigma_{33}=0$. This leads to plate models that are sometimes called solid plate models. The simplest one we will consider is built on the Mindlin-Reissner kinematic assumptions. According to the three-dimensional Hooke's law, we obtain for a compressible material

$$
\begin{aligned}
\sigma_{\alpha \beta}(u) & =\frac{E}{1+\nu}\left[\gamma_{\alpha \beta}(\underline{u})+x_{3} \gamma_{\alpha \beta}(\theta)+\frac{\nu}{1-2 \nu}\left[\gamma_{\eta \eta}(\underline{u})+x_{3} \gamma_{\eta \eta}(\theta)\right] \delta_{\alpha \beta}\right], \\
\sigma_{\alpha 3}(u) & =\frac{E}{2(1+\nu)}\left(\partial_{\alpha} u_{3}+\theta_{\alpha}\right), \\
\sigma_{33}(u) & =\frac{E \nu}{(1+\nu)(1-2 \nu)}\left[\gamma_{\eta \eta}(\underline{u})+x_{3} \gamma_{\eta \eta}(\theta)\right],
\end{aligned}
$$

and the linearized strain tensor field is still given by

$$
\gamma_{\alpha \beta}(u)=\frac{1}{2}\left(\partial_{\alpha} u_{\beta}+\partial_{\beta} u_{\alpha}\right)=\gamma_{\alpha \beta}(\underline{u})+x_{3} \gamma_{\alpha \beta}(\theta) \quad \text { and } \quad \gamma_{\alpha 3}=\frac{1}{2}\left(\partial_{\alpha} u_{3}+\theta_{\alpha}\right) .
$$

The membrane stress resultants $\left(N_{\alpha \beta}\right)$, the bending moments stress resultants $\left(M_{\alpha \beta}\right)$ and the shear stress resultants $\left(T_{\beta}\right)$ have close but not identical expression compared to the Mindlin-Reissner model:

$$
\begin{aligned}
N_{\alpha \beta} & =\frac{2 \varepsilon E}{(1+\nu)(1-2 \nu)}\left[(1-2 \nu) \gamma_{\alpha \beta}(\underline{u})+\nu \gamma_{\eta \eta}(\underline{u}) \delta_{\alpha \beta}\right], \\
M_{\alpha \beta} & =\frac{2 \varepsilon^{3} E}{3(1+\nu)(1-2 \nu)}\left[(1-2 \nu) \gamma_{\alpha \beta}(\theta)+\nu \gamma_{\eta \eta}(\theta) \delta_{\alpha \beta}\right], \\
T_{\beta} & =\frac{\varepsilon E}{1+\nu}\left[\partial_{\beta} u_{3}+\theta_{\beta}\right] .
\end{aligned}
$$

The non-penetration condition terms from (2.9) becomes

$$
\begin{aligned}
{\left[\sigma_{n}(u)+r\left(g-u_{n}\right)\right]_{-} } & =\left[\sigma_{i j}(u) n_{j} \bar{n}_{i}+r\left(g-\underline{u}_{\alpha} \bar{n}_{\alpha}-\varepsilon \theta_{\alpha} \bar{n}_{\alpha}-u_{3} \bar{n}_{3}\right)\right]_{-} \\
r v_{n}-\mathcal{O} \sigma_{n}(v) & =r\left(\underline{v}_{\alpha} \bar{n}_{\alpha}+\varepsilon \psi_{\alpha} \bar{n}_{\alpha}+v_{3} \bar{n}_{3}\right)-\mathcal{O} \sigma_{i j}(v) n_{j} \bar{n}_{i} .
\end{aligned}
$$

The term in the friction condition (2.10) reads now

$$
\sigma_{t}(u)-r u_{t}=\sigma_{i j}(u) n_{j} e_{i}-\left(\sigma_{i j}(u) n_{j} \bar{n}_{i}\right) \bar{n}-r\left(u-u_{n} \bar{n}\right),
$$

and the simplifications coming from $n=-e_{3}$ lead to

$$
\begin{aligned}
{\left[\sigma_{n}(u)+r\left(g-u_{n}\right)\right]_{-} } & =\left[-\sigma_{i 3}(u) \bar{n}_{i}+r\left(g-\underline{u}_{\alpha} \bar{n}_{\alpha}-\varepsilon \theta_{\alpha} \bar{n}_{\alpha}-u_{3} \bar{n}_{3}\right)\right]_{-}, \\
r v_{n}-\mathcal{O} \sigma_{n}(v) & =r\left(\underline{v}_{\alpha} \bar{n}_{\alpha}+\varepsilon \psi_{\alpha} \bar{n}_{\alpha}+v_{3} \bar{n}_{3}\right)+\mathcal{O} \sigma_{i 3}(v) \bar{n}_{i}, \\
\sigma_{t}(u)-r u_{t} & =\sigma_{i 3}(u)\left(\bar{n}_{i} \bar{n}-e_{i}\right)-r u_{t} .
\end{aligned}
$$




\section{Special case $\bar{n}=n=-e_{3}$}

In the special case $\bar{n}=n=-e_{3}$, we obtain:

$$
\begin{aligned}
{\left[\sigma_{n}(u)+r\left(g-u_{n}\right)\right]_{-} } & =\left[\sigma_{33}(u)+r\left(g+\underline{u}_{3}\right)\right]_{-} \\
& =\left[\frac{E \nu}{(1+\nu)(1-2 \nu)}\left[\gamma_{\eta \eta}(\underline{u})-\varepsilon \gamma_{\eta \eta}(\theta)\right]+r\left(g+\underline{u}_{3}\right)\right]_{-}, \\
r v_{n}-\mathcal{O} \sigma_{n}(v) & =-r \underline{v}_{3}-\mathcal{O} \sigma_{33}(v), \\
& =-r \underline{v}_{3}-\frac{\mathcal{O} E \nu}{(1+\nu)(1-2 \nu)}\left[\gamma_{\eta \eta}(\underline{v})-\varepsilon \gamma_{\eta \eta}(\psi)\right], \\
\sigma_{t}(u)-r u_{t} & =-\sigma_{\alpha 3}(u) e_{\alpha}-r u_{t}=-\frac{E}{2(1+\nu)}\left(\partial_{\alpha} \underline{u}_{3}+\theta_{\alpha}\right) e_{\alpha}-r\left(\underline{u}_{\alpha} e_{\alpha}+\varepsilon \theta_{\alpha} e_{\alpha}\right), \\
r v_{t}-\mathcal{O} \sigma_{t}(v) & =r v_{t}+\mathcal{O} \sigma_{\alpha 3}(v) e_{\alpha}=r\left(\underline{v}_{\alpha} e_{\alpha}+\varepsilon \psi_{\alpha} e_{\alpha}\right)+\mathcal{O} \frac{E}{2(1+\nu)}\left(\partial_{\alpha} \underline{v}_{3}+\psi_{\alpha}\right) e_{\alpha} .
\end{aligned}
$$

We then end up with the following formulation in this special case:

$$
\left\{\begin{array}{l}
\text { Find }(\underline{u}, \theta) \in \mathbb{V}_{\mathrm{MR}} \text { such that for all }(\underline{v}, \psi) \in \mathbb{V}_{\mathrm{MR}} \\
\int_{\Omega} N_{\alpha \beta} \gamma_{\alpha \beta}(\underline{v})+M_{\alpha \beta} \gamma_{\alpha \beta}(\psi)+T_{\beta}\left(\partial_{\beta} \underline{v}_{3}+\psi_{\beta}\right) \mathrm{d} \Omega \\
\quad-\frac{1}{r} \int_{\Omega}\left[\sigma_{33}(u)+r\left(g+\underline{u}_{3}\right)\right]_{-}\left(\mathcal{O} \sigma_{33}(v)+r \underline{v}_{3}\right) \mathrm{d} \Omega \\
\quad+\frac{1}{r} \int_{\Omega} \mathbf{P}_{B\left(0, \mathcal{F}\left[\sigma_{33}(u)+r\left(g+\underline{u}_{3}\right)\right]_{-}\right)}\left(\sigma_{\alpha 3}(u) e_{\alpha}+r u_{t}\right) \cdot\left(r v_{t}+\mathcal{O} \sigma_{\alpha 3}(v) e_{\alpha}\right) \mathrm{d} \Omega \\
\quad-\frac{\mathcal{O}}{r} \int_{\Omega} \sigma_{33}(u) \sigma_{33}(v)+\sigma_{\alpha 3}(u) \sigma_{\alpha 3}(v) \mathrm{d} \Omega=\int_{\Omega} F_{i} v_{i}-G_{\alpha} \psi_{\alpha} \mathrm{d} \Omega .
\end{array}\right.
$$

We note the presence of the pinch stress $\sigma_{33}(u)$ into the contact term, which means that the contact condition do not reduce to a pure penalty term. However, the pinch stress is proportional to $\gamma_{\eta \eta}(u)$, the divergence of $u$ and this only represent a coupling with the Poisson effect. This is due to the fact that no pinch deformation is considered with the Mindlin-Reissner kinematics.

\subsection{Weak formulation under solid plate displacements assumptions}

Now we focus our attention on the richest kinematics (1.1) that we insert into (2.11) and obtain, as previously, our Nitsche-based formulation for frictional contact.

The work of elastic forces reads 


$$
\begin{aligned}
& \int_{\Omega^{\varepsilon}} \sigma(u): \gamma(v) \mathrm{d} \Omega=\int_{\Omega}[\underbrace{\left(\int_{-\varepsilon}^{\varepsilon} \sigma_{\alpha \beta}(u) \mathrm{d} x_{3}\right)}_{N_{\alpha \beta}} \gamma_{\alpha \beta}(\underline{v})+\underbrace{\left(\int_{-\varepsilon}^{\varepsilon} x_{3} \sigma_{\alpha \beta}(u) \mathrm{d} x_{3}\right)}_{M_{\alpha \beta}} \gamma_{\alpha \beta}(\psi)] \mathrm{d} \Omega \\
& +\int_{\Omega}[\underbrace{\left(\int_{-\varepsilon}^{\varepsilon} \sigma_{\beta 3}(u) \mathrm{d} x_{3}\right)}_{T_{\beta}}\left(\partial_{\beta} \underline{v}_{3}+\psi_{\beta}\right)] \mathrm{d} \Omega \\
& +\int_{\Omega}[\underbrace{\left(\int_{-\varepsilon}^{\varepsilon} x_{3} \sigma_{\beta 3}(u) \mathrm{d} x_{3}\right)}_{Q_{\beta}} \partial_{\beta} \psi_{3}+\underbrace{\left(\int_{-\varepsilon}^{\varepsilon} \sigma_{33}(u) \mathrm{d} x_{3}\right)}_{P} \psi_{3}] \mathrm{d} \Omega .
\end{aligned}
$$

Taking into account (1.1) the linear approximation of the three-dimensional displacements in $\Omega^{\varepsilon}$, isotropic Hooke's law is given by

$$
\begin{aligned}
\sigma_{\alpha \beta}(u) & =\frac{E}{1+\nu}\left[\gamma_{\alpha \beta}(u)+\frac{\nu}{1-2 \nu} \gamma_{k k}(u) \delta_{\alpha \beta}\right], \\
\sigma_{\alpha 3}(u) & =\frac{E}{1+\nu} \gamma_{\alpha 3}(u) \\
\sigma_{33}(u) & =\frac{E}{1+\nu}\left[\gamma_{33}(u)+\frac{\nu}{1-2 \nu} \gamma_{k k}(u)\right]
\end{aligned}
$$

with the linearized strain tensor field

$$
\gamma_{\alpha \beta}(u)=\gamma_{\alpha \beta}(\underline{u})+x_{3} \gamma_{\alpha \beta}(\theta), \quad \gamma_{\alpha 3}(u)=\frac{1}{2}\left(\partial_{\alpha} \underline{u}_{3}+x_{3} \partial_{\alpha} \theta_{3}+\theta_{\alpha}\right), \quad \gamma_{33}(u)=\theta_{3},
$$

and we get

$$
\begin{aligned}
\sigma_{\alpha \beta}(u) & =\frac{E}{1+\nu}\left[\gamma_{\alpha \beta}(\underline{u})+x_{3} \gamma_{\alpha \beta}(\theta)+\frac{\nu}{1-2 \nu}\left[\gamma_{\eta \eta}(\underline{u})+x_{3} \gamma_{\eta \eta}(\theta)+\theta_{3}\right] \delta_{\alpha \beta}\right], \\
\sigma_{\alpha 3}(u) & =\frac{E}{2(1+\nu)}\left(\partial_{\alpha} u_{3}+x_{3} \partial_{\alpha} \theta_{3}+\theta_{\alpha}\right), \\
\sigma_{33}(u) & =\frac{E}{1+\nu}\left[\theta_{3}+\frac{\nu}{1-2 \nu}\left[\gamma_{\eta \eta}(\underline{u})+x_{3} \gamma_{\eta \eta}(\theta)+\theta_{3}\right]\right] .
\end{aligned}
$$

The expressions of $N_{\alpha \beta}, M_{\alpha \beta}, T_{\alpha}$ and $P$ are given by

$$
\begin{aligned}
N_{\alpha \beta} & =\frac{2 \varepsilon E}{1+\nu}\left[\gamma_{\alpha \beta}(\underline{u})+\frac{\nu}{1-2 \nu}\left(\gamma_{\eta \eta}(\underline{u})+\theta_{3}\right) \delta_{\alpha \beta}\right], \\
M_{\alpha \beta} & =\frac{2 \varepsilon^{3} E}{3(1+\nu)}\left[\gamma_{\alpha \beta}(\theta)+\frac{\nu}{1-2 \nu} \gamma_{\eta \eta}(\theta) \delta_{\alpha \beta}\right], \\
T_{\beta} & =\frac{\varepsilon E}{1+\nu}\left[\partial_{\beta} \underline{u}_{3}+\theta_{\beta}\right], \\
Q_{\beta} & =\frac{\varepsilon^{3} E}{3(1+\nu)} \partial_{\beta} \theta_{3}, \\
P & =\frac{2 \varepsilon E}{1+\nu}\left[\theta_{3}+\frac{\nu}{1-2 \nu}\left(\gamma_{\eta \eta}(\underline{u})+\theta_{3}\right)\right] .
\end{aligned}
$$


The non-penetration condition terms from (2.9) reads

$$
\begin{aligned}
{\left[\sigma_{n}(u)+r\left(g-u_{n}\right)\right]_{-} } & =\left[\sigma_{i j}(u) n_{j} \bar{n}_{i}+r\left(g-\underline{u}_{i} \bar{n}_{i}+\varepsilon \theta_{i} \bar{n}_{i}\right)\right]_{-} \\
r v_{n}-\mathcal{O} \sigma_{n}(v) & =r\left(\underline{v}_{i} \bar{n}_{i}-\varepsilon \psi_{i} \bar{n}_{i}\right)-\mathcal{O} \sigma_{i j}(v) n_{j} \bar{n}_{i} .
\end{aligned}
$$

The term in the friction condition (2.10) is still

$$
\sigma_{t}(u)-r u_{t}=\sigma_{i j}(u) n_{j} e_{i}-\left(\sigma_{i j}(u) n_{j} \bar{n}_{i}\right) \bar{n}-r\left(u-u_{n} \bar{n}\right),
$$

and the simplifications coming form $n=-e_{3}$ leads to

$$
\begin{aligned}
{\left[\sigma_{n}(u)+r\left(g-u_{n}\right)\right]_{-} } & =\left[-\sigma_{i 3}(u) \bar{n}_{i}+r\left(g-\underline{u}_{i} \bar{n}_{i}+\varepsilon \theta_{i} \bar{n}_{i}\right)\right]_{-}, \\
r v_{n}-\mathcal{O} \sigma_{n}(v) & =r\left(\underline{v}_{i} \bar{n}_{i}-\varepsilon \psi_{i} \bar{n}_{i}\right)+\mathcal{O} \sigma_{i 3}(v) \bar{n}_{i}, \\
\sigma_{t}(u)-r u_{t} & =\sigma_{i 3}(u)\left(\bar{n}_{i} \bar{n}-e_{i}\right)-r u_{t} .
\end{aligned}
$$

\section{Special case $\bar{n}=n=-e_{3}$}

In the special case $\bar{n}=n=-e_{3}$, we obtain:

$$
\begin{aligned}
{\left[\sigma_{n}(u)+r\left(g-u_{n}\right)\right]_{-} } & =\left[\sigma_{33}(u)+r\left(g+\underline{u}_{3}-\varepsilon \theta_{3}\right)\right]_{-}, \\
& =\left[\frac{E}{1+\nu}\left[\theta_{3}+\frac{\nu}{1-2 \nu}\left[\gamma_{\eta \eta}(\underline{u})-\varepsilon \gamma_{\eta \eta}(\theta)+\theta_{3}\right]\right]+r\left(g+\underline{u}_{3}-\varepsilon \theta_{3}\right)\right]_{-}, \\
r v_{n}-\mathcal{O} \sigma_{n}(v) & =-r\left(\underline{v}_{3}-\varepsilon \psi_{3}\right)-\mathcal{O} \sigma_{33}(v), \\
& =-r\left(\underline{v}_{3}-\varepsilon \psi_{3}\right)-\frac{\mathcal{O} E}{1+\nu}\left[\psi_{3}+\frac{\nu}{1-2 \nu}\left[\gamma_{\eta \eta}(\underline{v})-\varepsilon \gamma_{\eta \eta}(\psi)+\psi_{3}\right]\right], \\
\sigma_{t}(u)-r u_{t} & =-\sigma_{\alpha 3}(u) e_{\alpha}-r u_{t} \\
& =-\frac{E}{2(1+\nu)}\left(\partial_{\alpha} \underline{u}_{3}-\varepsilon \partial_{\alpha} \theta_{3}+\theta_{\alpha}\right) e_{\alpha}-r\left(\underline{u}_{\alpha} e_{\alpha}+\varepsilon \theta_{\alpha} e_{\alpha}\right), \\
r v_{t}-\mathcal{O} \sigma_{t}(v) & =r v_{t}+\mathcal{O} \sigma_{\alpha 3}(v) e_{\alpha} \\
& =r\left(\underline{v}_{\alpha} e_{\alpha}+\varepsilon \psi_{\alpha} e_{\alpha}\right)+\frac{\mathcal{O} E}{2(1+\nu)}\left(\partial_{\alpha} \underline{v}_{3}-\varepsilon \partial_{\alpha} \psi_{3}+\psi_{\alpha}\right) e_{\alpha} .
\end{aligned}
$$

Finally, denoting

$$
\mathbb{V}_{\mathrm{SP}}:=\left\{(\underline{v}, \psi) \in H^{1}\left(\Omega ; \mathbb{R}^{3}\right) \times H^{1}\left(\Omega ; \mathbb{R}^{3}\right) \mid \underline{v}=0 \quad \text { on } \quad \Gamma_{D}, \quad \psi=0 \quad \text { on } \quad \Gamma_{D}\right\},
$$

and defining additionally $G_{3}:=\int_{-\varepsilon}^{\varepsilon} x_{3} f_{3}^{V} \mathrm{~d} x_{3}+\varepsilon \ell_{3}$, the formulation of the solid plate model with Nitsche's contact reads in this case: 


$$
\left\{\begin{array}{l}
\text { Find }(\underline{u}, \theta) \in \mathbb{V}_{\mathrm{SP}} \text { such that for all }(\underline{v}, \psi) \in \mathbb{V}_{\mathrm{SP}} \\
\int_{\Omega} N_{\alpha \beta} \gamma_{\alpha \beta}(\underline{v})+M_{\alpha \beta} \gamma_{\alpha \beta}(\psi)+T_{\beta}\left(\partial_{\beta} \underline{v}_{3}+\psi_{\beta}\right)+Q_{\beta} \partial_{\beta} \psi_{3}+P \psi_{3} \mathrm{~d} \Omega \\
\quad-\frac{1}{r} \int_{\Omega}\left[\sigma_{33}(u)+r\left(g+\underline{u}_{3}-\varepsilon \theta_{3}\right)\right]_{-}\left(\mathcal{O} \sigma_{33}(v)+r \underline{v}_{3}-\varepsilon \psi_{3}\right) \mathrm{d} \Omega \\
\quad+\frac{1}{r} \int_{\Omega} \mathbf{P}_{B\left(0, \mathcal{F}\left[\sigma_{33}(u)+r\left(g+\underline{u}_{3}-\varepsilon \theta_{3}\right)\right]_{-}\right)}\left(\sigma_{\alpha 3}(u) e_{\alpha}+r u_{t}\right) \cdot\left(r v_{t}+\mathcal{O} \sigma_{\alpha 3}(v) e_{\alpha}\right) \mathrm{d} \Omega \\
-\frac{\mathcal{O}}{r} \int_{\Omega} \sigma_{33}(u) \sigma_{33}(v)+\sigma_{\alpha 3}(u) \sigma_{\alpha 3}(v) \mathrm{d} \Omega=\int_{\Omega} F_{i} v_{i}-G_{i} \psi_{i} \mathrm{~d} \Omega .
\end{array}\right.
$$

As expected, we see that compared to the previous plate models, the solid plate model, due to its enriched transverse displacement, shows both pinch stress and pinch deformation in contact Nitsche terms.

\section{Gustafsson, Stenberg and Videman discrete Nitsche's formulation of the KIRCHHOFF-LOVE PLATE MODEL}

In this section, we describe the Nitsche formulation of the Kirchhoff-Love plate model constrained by a rigid obstacle introduced in [24]. For simplicity, only the transverse displacement variable $\left(\tilde{u}:=u_{3}: \Omega \longrightarrow \mathbb{R}\right)$ is considered. We address this formulation mainly to compare numerical results we obtain below with the models proposed previously. The variational formulation for a Kirchhoff-Love thin elastic clamped/free plate made of a homogeneous and isotropic material and lying over a rigid obstacle, consists in the following variational inequality:

$$
\left\{\begin{array}{l}
\text { Find }\left(\tilde{u}, \tilde{\sigma}_{n}\right) \in \tilde{\mathbb{V}} \times \Lambda \text { such that for any }(\tilde{w}, \mu) \in \tilde{\mathbb{V}} \times \Lambda \\
\int_{\Omega} D\left[(1-\nu) \partial_{\alpha \beta}^{2} \tilde{u}+n \Delta \tilde{u} \delta_{\alpha \beta}\right] \partial_{\alpha \beta}^{2} \tilde{w} \mathrm{~d} \Omega-\left\langle\tilde{\sigma}_{n}, \tilde{w}\right\rangle_{\tilde{\mathbb{V}}^{\prime}, \tilde{\mathbb{V}}}=\int_{\Omega} \tilde{f} \tilde{w} \mathrm{~d} \Omega, \\
\left\langle\mu-\tilde{\sigma}_{n}, \tilde{w}\right\rangle_{\tilde{\mathbb{V}}^{\prime}, \tilde{\mathbb{V}}} \geq 0
\end{array}\right.
$$

with $\tilde{\sigma}_{n}$ the Lagrange multiplier for the contact condition (the contact reaction), $\tilde{f}:=\int_{-\varepsilon}^{\varepsilon} f_{3}^{V} \mathrm{~d} x_{3}$ the resulting transverse loading and the bending modulus $D:=\frac{2 E \varepsilon^{3}}{3\left(1-\nu^{2}\right)}$ the bending modulus, $\varepsilon>0$ being assumed to be constant all along the plate. The plate is assumed to be clamped on a part $\Gamma_{D}$ of the boundary $\partial \Omega$. Then, the space for the displacements is

$$
\tilde{\mathbb{V}}:=\left\{\tilde{w} \in H^{2}(\Omega) \mid \tilde{w}(x)=\partial_{n} \tilde{w}(x)=0, \quad \forall x \in \Gamma_{D}\right\},
$$

where $\partial_{n} \tilde{w}$ is the normal derivative along $\Gamma_{D}$, and the convex of admissible Lagrange multipliers is

$$
\Lambda:=\left\{\mu \in \tilde{\mathbb{V}}^{\prime} \mid\langle\mu, \tilde{w}\rangle_{\tilde{\mathbb{V}}^{\prime}, \tilde{\mathbb{V}}} \geq 0, \quad \forall w \in \tilde{\mathbb{V}}, w \leq 0\right\} .
$$

The well-posedness of (5.1) is guaranteed by the following result [32].

Lemma 5.1. The bilinear form $\tilde{a}: \tilde{\mathbb{V}} \times \tilde{\mathbb{V}} \rightarrow \mathbb{R}$ defined by

$$
\tilde{a}(\tilde{u}, \tilde{v}):=\int_{\Omega} D\left[(1-\nu) \partial_{\alpha \beta}^{2} \tilde{u}+\nu \Delta \tilde{u} \delta_{\alpha \beta}\right] \partial_{\alpha \beta}^{2} \tilde{v} \mathrm{~d} \Omega,
$$

is a scalar product on $\tilde{\mathbb{V}}$ which is equivalent to the canonical scalar product of $H^{2}(\Omega)$ defined on $\tilde{\mathbb{V}}$. 
The contact reaction $\tilde{\sigma}_{n}(u)=D \Delta^{2} \tilde{u}-\tilde{f}$ is a positive measure, possibly singular, and its support included in the contact zone, denoted $\Omega_{C}$. For more details see [31].

The reaction force between the obstacle and the plate can be approximated by

$$
\tilde{\sigma}_{n}^{k}=-\frac{1}{k}[\tilde{u}-g]_{+}
$$

where $k>0$ is the penalty coefficient. Classically, in the limit $k \rightarrow 0, \tilde{\sigma}_{n}^{k}$ tends to $\tilde{\sigma}_{n}(u)$, which means that the obstacle becomes rigid and the problem is reduced to that of constrained minimization (5.1).

Now, we summarize the technique proposed in [24]. This approach is analogous to the derivation of Nitsche's method for 3D-contact problems. Let $\tilde{\mathcal{T}}_{h}$ be a conforming shape regular triangulation of $\Omega$ which we assume to be polygonal, and the associated finite element subspace is $\widetilde{\mathbb{V}}^{h} \subset \widetilde{\mathbb{V}}$. Optimality is achieved when the Lagrange multiplier is approximated with four degrees smaller polynomials than that of the displacement variable, for example, when the Argyris element is coupled with a piecewise linear and discontinuous approximation of the Lagrange multiplier.

A reformulation of the normal contact conditions with a Nitsche-based method consists in giving an explicit expression of the discrete Lagrange multiplier from the previous formulation of contact problem (5.1). As mentioned above, the contact reaction is a possibly singular measure, therefore the following $L^{2}$-approximation of the reaction force is considered in [24]:

$$
\left.\tilde{\sigma}_{n}^{\pi^{h}}(u)\right|_{K}:=\frac{1}{k+\tilde{r} h_{K}^{4}}\left[\left.\pi^{h}(g)\right|_{K}-\left.\pi^{h}\left(\tilde{u}^{h}\right)\right|_{K}+\tilde{r} h_{K}^{4}\left(\pi^{h}\left(\Delta^{2} \tilde{u}^{h}\right)-\pi^{h}(\tilde{f})\right)\right]_{+} \quad \forall K \in \tilde{\mathcal{T}}_{h},
$$

where $\pi^{h}$ is the $L^{2}$-projection on $\tilde{\mathbb{V}}^{h}, k \geq 0$ is the penalty coefficient from (5.2) and $\tilde{r} \geq 0$ is the stabilization or Nitsche's parameter. This hybrid formulation (5.3) of the Lagrange multiplier corresponds to a pure penalty approach when $\tilde{r}=0$ and a pure Nitsche's method if $k=0$.

We introduce the function $H: \Omega \longrightarrow \mathbb{R}$ such that $\left.H\right|_{K}=h_{K}$, for all $K \in \tilde{\mathcal{T}}_{h}$. A finite element approximation of (5.1) based on hybrid Nitsche's method (5.3) is:

$$
\left\{\begin{array}{l}
\text { Find } \tilde{u}^{h} \in \tilde{\mathbb{V}}^{h} \text { such that } \\
\tilde{a}^{h}\left(\tilde{u}^{h}, \tilde{v}^{h} ; \tilde{u}^{h}\right)=\tilde{L}^{h}\left(\tilde{v}^{h} ; \tilde{u}^{h}\right) \quad \forall \tilde{v}^{h} \in \tilde{\mathbb{V}}^{h}
\end{array}\right.
$$

where

$$
\begin{aligned}
\tilde{a}^{h}\left(\tilde{u}^{h}, \tilde{v}^{h} ; \tilde{u}^{h}\right):= & \tilde{a}\left(\tilde{u}^{h}, \tilde{v}^{h}\right)+\int_{\Omega_{C}\left(\tilde{w}^{h}\right)} \frac{1}{k+\tilde{r} H^{4}} \tilde{u}^{h} \tilde{v}^{h} \mathrm{~d} \Omega-\int_{\Omega_{C}\left(\tilde{w}^{h}\right)} \frac{\tilde{r} H^{4}}{k+\tilde{r} H^{4}} D \Delta^{2}\left(\tilde{u}^{h}\right) \tilde{v}^{h} \mathrm{~d} \Omega \\
& -\int_{\Omega_{C}\left(\tilde{w}^{h}\right)} \frac{\tilde{r} H^{4}}{k+\tilde{r} H^{4}} D \Delta^{2}\left(\tilde{v}^{h}\right) \tilde{u}^{h} \mathrm{~d} \Omega-\int_{\Omega_{C}\left(\tilde{w}^{h}\right)} \frac{k \tilde{r} H^{4}}{k+\tilde{r} H^{4}} D^{2} \Delta^{2}\left(\tilde{u}^{h}\right) \Delta^{2} \tilde{v}^{h} \mathrm{~d} \Omega \\
& -\int_{\Omega \backslash \Omega_{C}\left(\tilde{w}^{h}\right)} \tilde{r} H^{4} D^{2} \Delta^{2}\left(\tilde{u}^{h}\right) \Delta^{2} \tilde{v}^{h} \mathrm{~d} \Omega, \\
\tilde{L}^{h}\left(\tilde{v}^{h} ; \tilde{u}^{h}\right):= & \int_{\Omega} \tilde{f} \tilde{v}^{h} \mathrm{~d} \Omega+\int_{\Omega_{C}\left(\tilde{w}^{h}\right)} \frac{1}{k+\tilde{r} H^{4}} g \tilde{v}^{h} \mathrm{~d} \Omega \\
& -\int_{\Omega_{C}\left(\tilde{w}^{h}\right)} \frac{\tilde{r} H^{4}}{k+\tilde{r} H^{4}} D \Delta^{2}\left(\tilde{v}^{h}\right) g \mathrm{~d} \Omega-\int_{\Omega_{C}\left(\tilde{w}^{h}\right)} \frac{\tilde{r} H^{4}}{k+\tilde{r} H^{4}} f \tilde{v}^{h} \mathrm{~d} \Omega \\
& -\int_{\Omega_{C}\left(\tilde{w}^{h}\right)} \frac{\tilde{r} k H^{4}}{k+\tilde{r} H^{4}} D \Delta^{2}\left(\tilde{v}^{h}\right) f \mathrm{~d} \Omega-\int_{\Omega \backslash \Omega_{C}\left(\tilde{w}^{h}\right)} \tilde{r} H^{4} D \tilde{f} \Delta^{2} \tilde{v}^{h} \mathrm{~d} \Omega .
\end{aligned}
$$




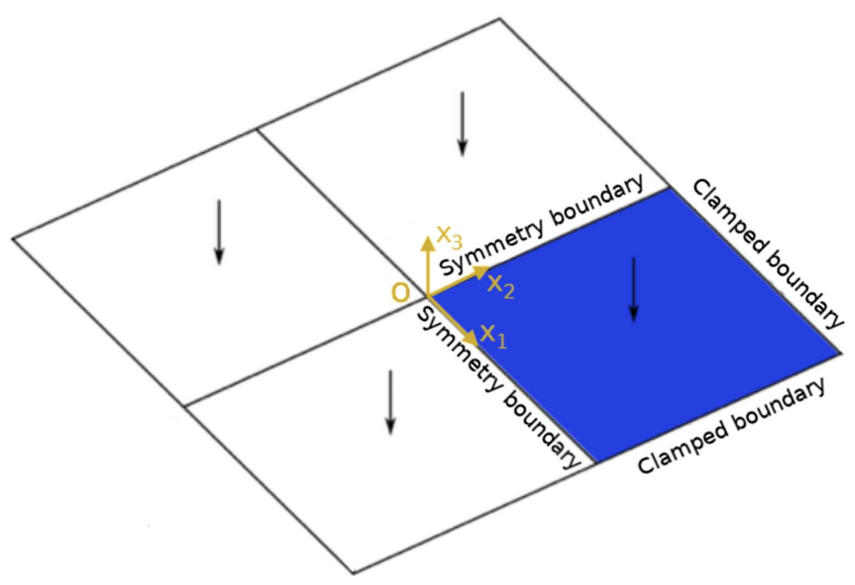

FiguRE 2. Square plate under transverse load, only a quarter of the plate (blue area) is modeled.

The contact set $\Omega_{C}\left(\tilde{w}^{h}\right)$ above is given by

$$
\Omega_{C}\left(\tilde{w}^{h}\right):=\left\{(x, y) \in \Omega \mid \tilde{\sigma}_{n}^{h}\left(\tilde{w}^{h}\right)>0\right\} .
$$

The practical solution algorithm for Problem (5.4) is a fixed point process where at each step the contact set $\Omega_{C}$ is approximated using the displacement field from the previous iteration so that System (5.4) becomes linear. The process is complete when the norm of the displacement field is below a predetermined tolerance. The stopping criterion is formulated with respect to the strain energy norm $\|w\|_{\tilde{a}}:=\sqrt{\tilde{a}(w, w)}$.

\section{Numerical RESUlts}

The numerical comparison of the different approaches is done on the frictionless contact between a rectangular elastic plate and two different rigid obstacles: a flat and a paraboloid one. The Kirchhoff-Love, Mindlin-Reissner and solid plate models are compared to a reference solution obtained directly from the 3D elasticity model and using an augmented Lagrangian multiplier technique to approximate the contact condition as in $[1,33]$. To avoid as much as possible the shear locking for the reference solution, quadratic elements ( $Q_{2}$-Lagrange elements) with two layers of elements in the thickness and a very fine mesh $(100 \times 100 \times 2$ elements $)$ are used. The main goal is then to determinate which model will be the closest to the $3 \mathrm{D}$ reference solution.

Numerical tests are performed on a domain $\left.\Omega^{\varepsilon}=\right]-10,10\left[{ }^{2} \times\right]-\varepsilon, \varepsilon[$, for different values of the thickness $\varepsilon$ and subjected to different distributed transverse loads $f$. The contact surface $\Gamma_{C}$ is still the lower part of $\Omega^{\varepsilon}$ (i.e. $\Gamma_{C}:=\Gamma_{-}^{\varepsilon}=\left\{x \in \partial \Omega^{\varepsilon}: x_{3}=-\varepsilon\right\}$ ). For the sake of simplicity, the plate is clamped along all its lateral sides. Because of the symmetry of this problem, only a quarter of the geometry is taken into account, where two of the lateral boundaries are clamped (see Fig. 2).

We assume that the plate is made of an isotropic elastic material with a Young modulus of $E=200 \mathrm{GPa}$ and a Poisson ratio $\nu=0.3$. Following the justification given in Section 7, Proposition 7.1, Nitsche's parameter is chosen equal to $r=\frac{E}{\varepsilon}$. Moreover, only the symmetric variant $\mathcal{O}=1$ is considered. The relative error is given by the ratio

$$
\frac{\left\|u^{h}-u^{\mathrm{ref}}\right\|}{\left\|u^{\mathrm{ref}}\right\|}
$$

with $L^{2}$-norm or $H^{1}$-semi-norm on $\Omega^{\varepsilon}$. This means in particular that the norms are applied on a 3D reconstruction of the $2 \mathrm{D}$ solutions obtained from the different plate models. 


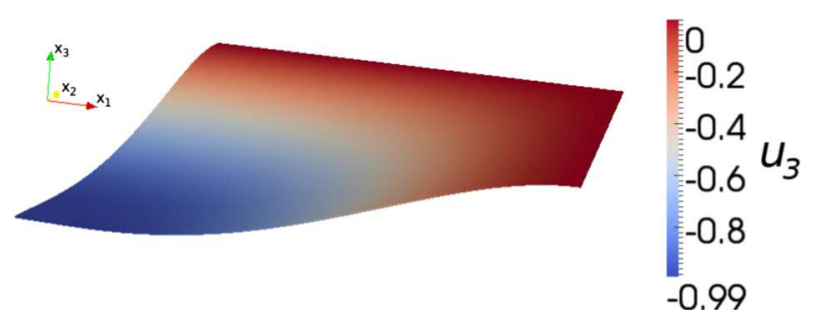

FIGURE 3 . Very thin square plate $(\varepsilon=0.01 \mathrm{~m})$ under uniform volume load and flat obstacle: $3 \mathrm{D}$ reference solution with the augmented Lagrangian method and $Q_{2}$-Lagrange elements.

The discrete contact problem is solved using a generalized Newton method on our open-source finite element library GetFEM++ (see [34] and http://getfem.org).

\subsection{Finite element approximation of the plate models}

The numerical convergence tests will be performed on a family of quadrangulations $\mathcal{T}_{h}$ of the mid-plane domain $\Omega$ such that $\Omega=\bigcup_{K \in \mathcal{T}_{h}} K$. We denote $h_{K}$ the diameter of $K \in \mathcal{T}_{h}$ and $h=\max _{K \in \mathcal{T}_{h}} h_{K}$.

For the Kirchhoff-Love and Mindlin-Reissner models, problems can be decoupled in a plane elasticity problem (membrane displacement) and a transverse displacement problem (bending problem). The membrane displacements are approximated with standard $Q_{1}$-Lagrange quadrilateral elements (also called QUAD4 element).

The bending problem of the Kirchhoff-Love model corresponds to a fourth-order partial differential equation. Thus, a conformal finite element approximation space must be a subspace of $H^{2}(\Omega)$ which requires the use of $\mathcal{C}^{1}$-finite elements $\left(\mathcal{C}^{0}\right.$-interior penalty method such as the one presented in [6] can be an interesting alternative). Among the available elements having this regularity (see [14]), we chose the reduced FVS (Fraeijs de VeubekeSanders) quadrangles for our numerical tests. The FVS element basis functions are piecewise $P_{3}$ polynomials on a division into four sub-triangles of the quadrilateral, with $\mathcal{C}^{1}$-continuity across each internal edge. In addition, to decrease the number of degrees of freedom, the normal derivative is assumed to vary linearly along the external edges of the elements (this assumption does not hold on the internal edges). Consequently, this element has the same computational cost in terms of degrees of freedom as the standard first-order elements used for Mindlin-Reissner bending problem (3 degrees of freedom per vertex, the deflection and the two section rotations).

For Mindlin-Reissner and solid plate models, the transverse displacement, the sections rotations and the pinching are approximated with $Q_{1}$-Lagrange quadrilateral elements. A very important issue is that this element is subject to the so-called shear locking so that a direct Galerkin procedure does not give a satisfactory approximation. There are several ways to circumvent the shear locking problem such as reduced integration and MITC projection. We use here the MITC projection (Mixed Interpolation of Tensorial Components) which was first developed to reduce shear locking for the 4-node continuum mechanics based MITC4 element [4]. The error analysis [3] performed on this element showed that the MITC4 element is optimally convergent for deflections and rotations on regular meshes and static analysis.

The solid plate model is subjected to a supplementary locking between pinching and membrane deformation due to the term

$$
\frac{2 \varepsilon \nu E}{(1+\nu)(1-2 \nu)}\left(\gamma_{\eta \eta}(\underline{u})+\theta_{3}\right)\left(\gamma_{\eta \eta}(\underline{v})+\psi_{3}\right),
$$

in the weak formulation (4.4). This supplementary locking is treated with a reduced integration technique, in the sense that a single Gauss point is used for this term. 


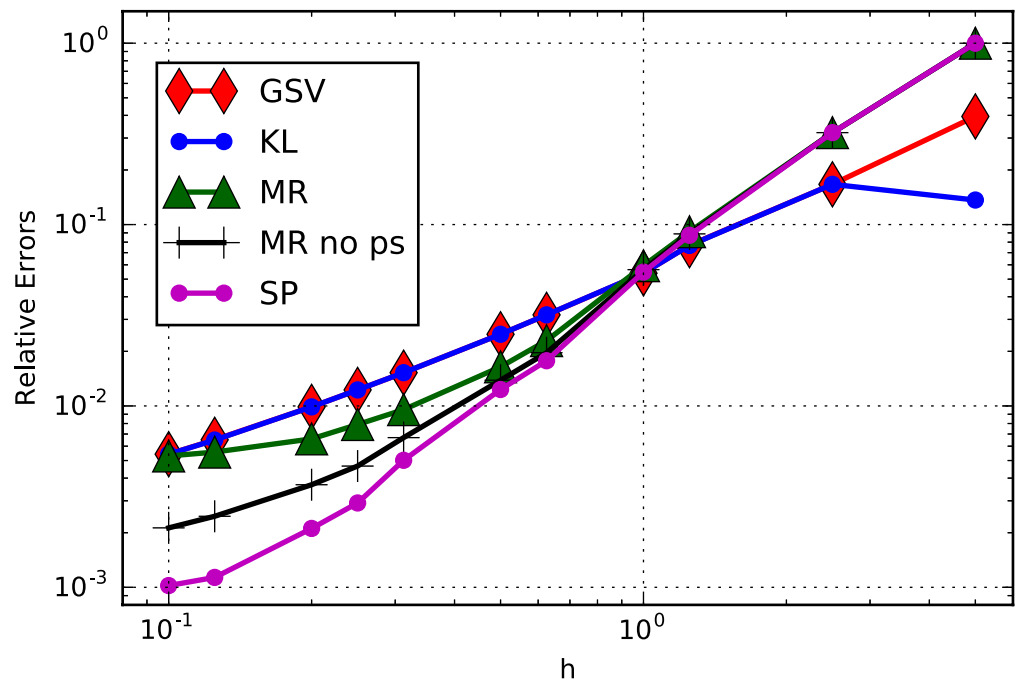

FiguRE 4. Relative $L^{2}\left(\Omega^{\varepsilon}\right)$-norm of the error in displacement with respect to the mesh size for the different plate models, comparatively to the 3D solution. Very thin plate on a flat obstacle with a uniform volume load.

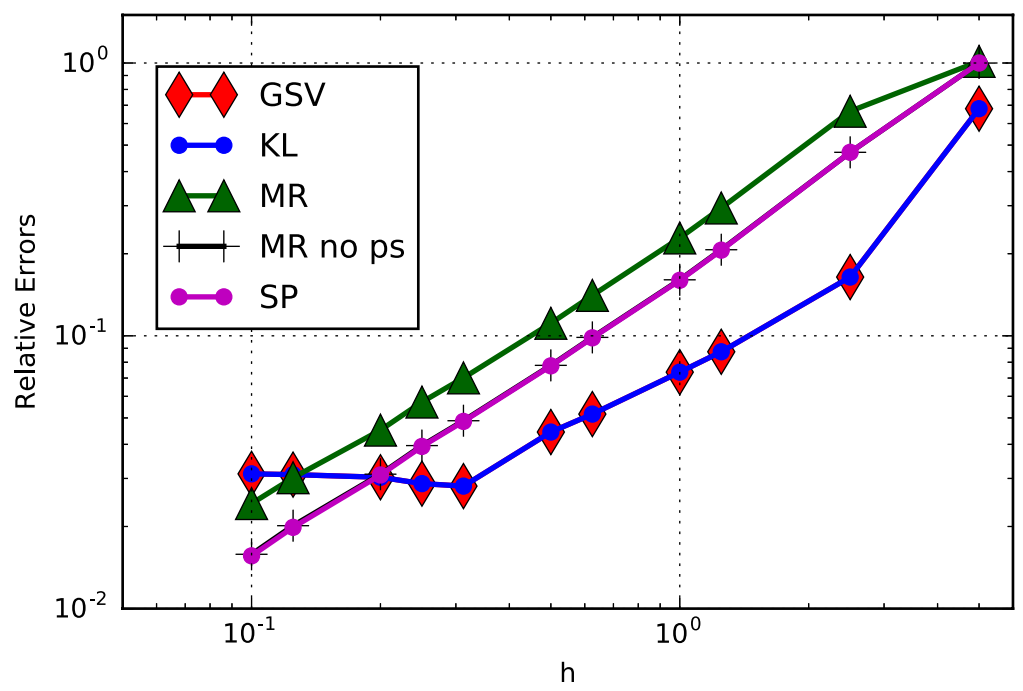

FiguRE 5. Relative $H^{1}\left(\Omega^{\varepsilon}\right)$-norm of the error in displacement with respect to the mesh size for the different plate models, comparatively to the 3D solution. Very thin plate on a flat obstacle with a uniform volume load.

\subsection{Numerical results for a very thin plate, a flat obstacle and a uniform volume load}

A very thin plate with $\varepsilon=0.01 \mathrm{~m}$ is first considered with a uniform volume load $f^{V}=-1 \mathrm{Mpa}$ and a flat obstacle represented by the initial gap $g=0.1-\varepsilon$. The reference 3D solution is represented in Figure 3 as an illustration.

The convergence test for the different models is represented on Figure 4 for the $L^{2}\left(\Omega^{\varepsilon}\right)$-norm of the displacement and on Figure 5 for the $H^{1}\left(\Omega^{\varepsilon}\right)$-semi-norm. In these figures, and in the rest of the paper, "GSV" refers to 


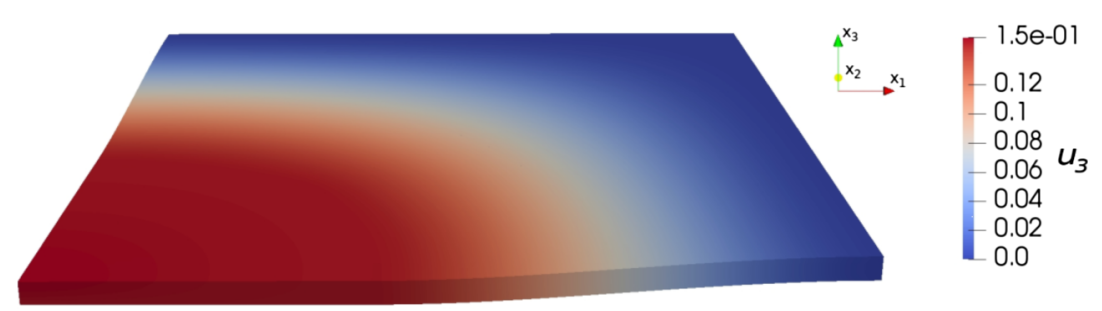

Figure 6. Thicker square plate $(\varepsilon=0.05 \mathrm{~m})$ under very large Gaussian volume load and flat obstacle: 3D reference solution with the augmented Lagrangian method and $Q_{2}$-Lagrange elements.

the method of Gustafsson, Stenberg and Videman (with the penalty coefficient $k=0$ ), "KL" refers to the model with Kirchhoff-Love assumptions of Section 3.1, "MR" to the model with the Mindlin-Reissner assumptions of Section 3.2, "MR no ps" to the model of Section 4.1 and "SP" to the solid plate model of Section 4.2.

As the plate is very thin, the plate models give some good approximation of the 3D solution even for small mesh sizes. This results in a decrease in error for small mesh sizes. One can see that the curves start to slow down their decrease for $h=0.1$, which is the smallest mesh size used.

Kirchhoff-Love models are a little more accurate for coarse meshes but are outpaced by Mindlin-Reissner and solid plate models for finer meshes. For very fine meshes, the most accurate models are those that do not take into account the plane stress assumption ("MR no ps" and "SP").

\subsection{Numerical results for a thicker plate, a flat obstacle and a very large Gaussian volume load}

We now consider a thicker plate with $\varepsilon=0.05$, still with a flat obstacle defined by the gap $g=0.15$ and subjected to a very large Gaussian volume load $f^{V}(x)=-50000 \exp \left(-\left(x_{1}^{2}+x_{2}^{2}\right)\right)$ MPa. A large uniform load has not been considered because it concentrates the deformation close to the boundaries where the plate is clamped. The reference solution is plotted in Figure 6.

The convergence performances are studied for $L^{2}\left(\Omega^{\varepsilon}\right)$-norm in Figure 7 and for $H^{1}\left(\Omega^{\varepsilon}\right)$-semi-norm in Figure 8 . Of course, in this experiment and due to the very large load, the pinch deformation is more important. It is again observed on these curves that the solid plate model "SP" is the most accurate on very fine meshes.

An interesting comparison here is the one between the two models based on Kirchhoff-Love assumptions. The main difference is that in the "GSV" model with the penalty parameter $k=0$, the contact condition is prescribed by a Nitsche method which is equivalent to a stabilized Lagrange multiplier method with static condensation of the Lagrange multiplier. This of course guarantees a stricter contact condition than the "KL" model presented in Section 3.1 which corresponds to a penalized contact condition.

One possible interpretation on the fact that "KL" model is far more accurate that "GSV" model here is that the penalized contact condition with a penalty coefficient $E / \varepsilon$ close to the stiffness of the elastic material gives a more realistic contact condition in the sense that it better reflects the contribution of the pinching of the plate.

This interpretation is supported by the curves in Figures 9-11. On these figures, the transverse displacement is drawn close to the contact zone. The difference between the three figures is that the transverse displacement is taken respectively on the bottom of the plate (contact boundary $\Gamma_{-}^{\varepsilon}$ ), on the mid-plane and on the top of the plate respectively. Of course, for the models based on the Mindlin-Reissner or Kirchhoff-Love assumptions, the three curves are identical since the transverse displacement is constant across the thickness. A difference is visible only for the "SP" model and the reference solution. We can see the very good behavior of the "SP" model which is close to the reference solution on the three figures with practically no penetration on the contact boundary $\Gamma_{-}^{\varepsilon}$ (see Fig. 9), conversely to the other models. We can see also that most of the models give a good approximation of the deformation on the mid-plane, except the "GSV" model since it corresponds to a 


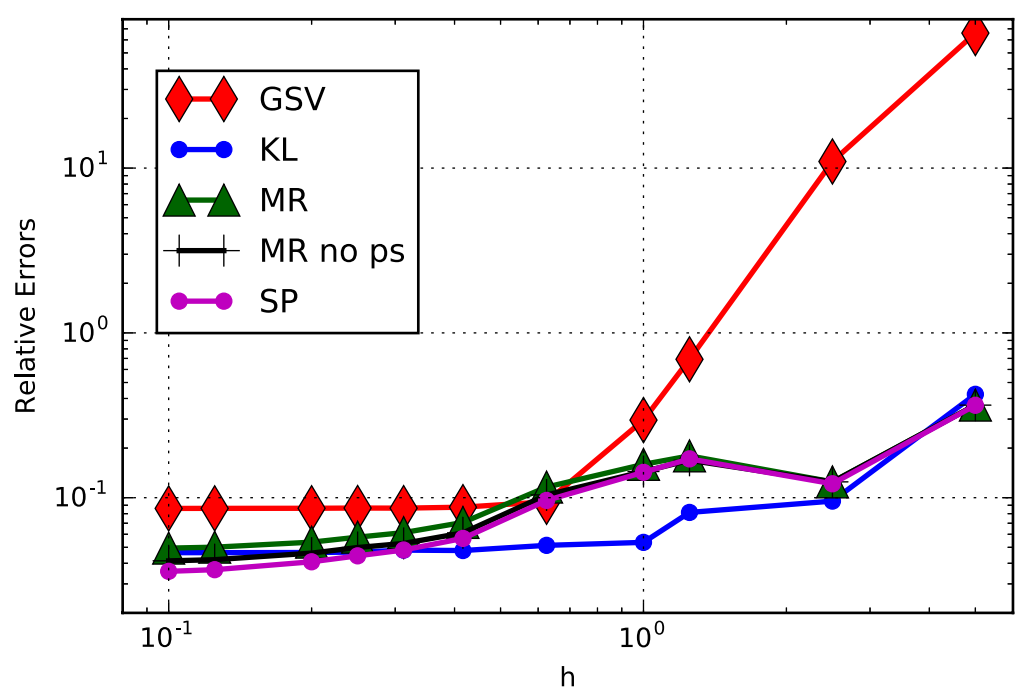

FiguRe 7. Relative $L^{2}\left(\Omega^{\varepsilon}\right)$-norm of the error in displacement with respect to the mesh size for the different plate models, comparatively to the 3D solution. Thicker plate on a flat obstacle with a Gaussian volume load.

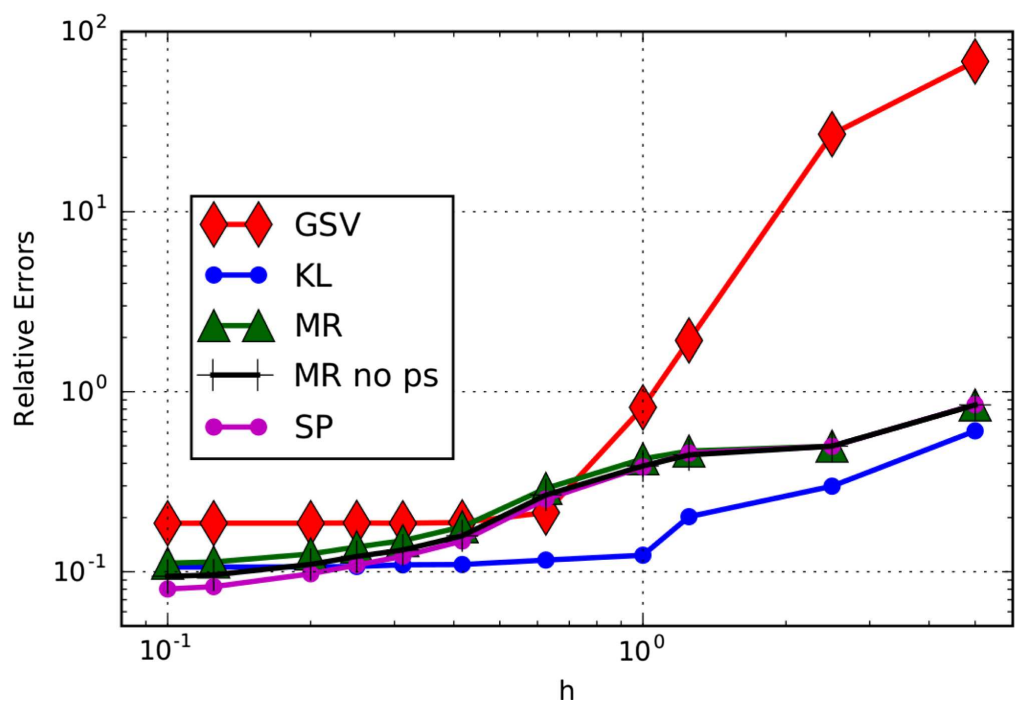

Figure 8. Relative $H^{1}\left(\Omega^{\varepsilon}\right)$-semi-norm of the error in displacement with respect to the mesh size for the different plate models, comparatively to the 3D solution. Thicker plate on a flat obstacle with a Gaussian volume load.

quasi-strict non-interpenetration condition when $k=0$. We can conclude that for an important pinching of the plate, the "SP" model leads to a more realistic approximation of the contact condition and conversely, the "GSV" is less accurate due to the more strict non-interpenetration condition. 


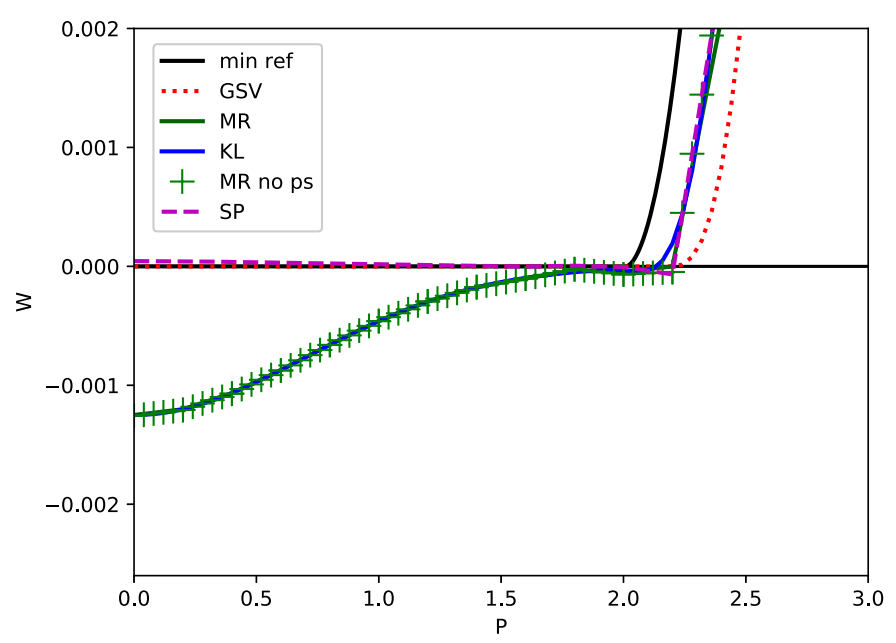

Figure 9. Lower-plane displacement on lateral edge: interpenetration with the rigid obstacle.

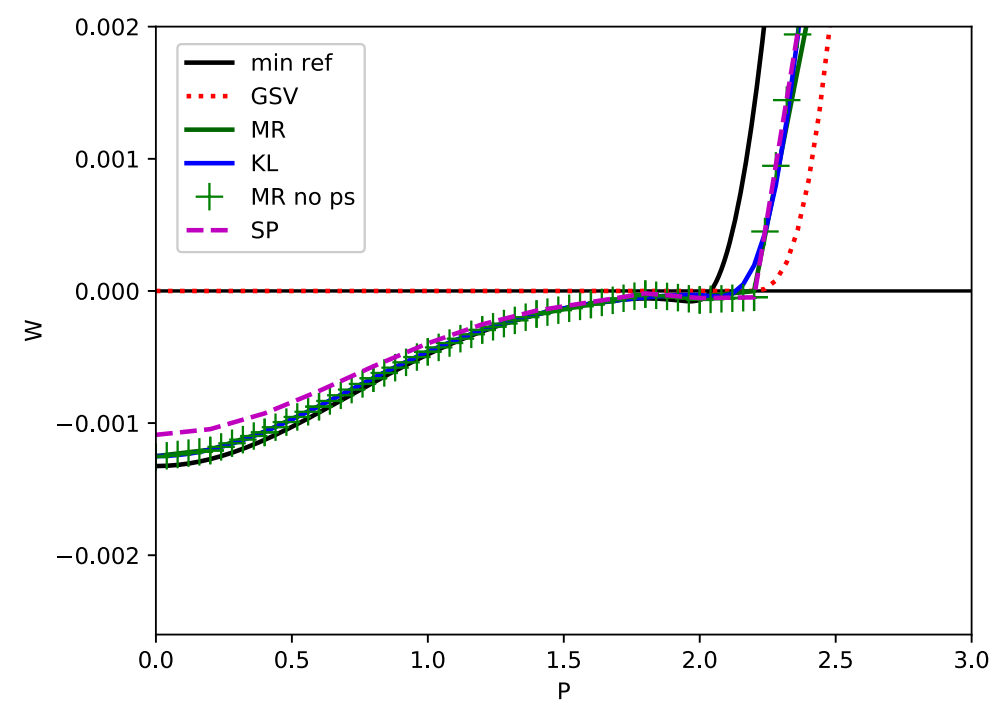

Figure 10. Mid-plane displacement on lateral edge.

\subsection{Numerical results with a paraboloid obstacle and no volume load}

For this last numerical case, we still consider a plate of medium thickness $\varepsilon=0.5$, on $\Omega=[-1,1] \times[-1,1]$, without load $\left(f^{V}=0\right)$, a paraboloid obstacle represented by the initial gap $g=x_{1}^{2}+x_{2}^{2}$ and a nonzero transverse displacement $u_{3}^{\varepsilon}=-0.4$ prescribed on the boundary $\Gamma_{D}^{\varepsilon}$.

The reference solution is shown in Figure 12. In that case, one would expect to be closer to a plane stress situation since the only force acting on the plate is the contact force which acting primarily at the frontier of the effective contact area.

The convergence test is shown in Figures 13 and 14. The numerical results are similar to those of the previous case with the flat obstacle. There is still a big difference between the "GSV" model and the "KL" ones although they are based on the same kinematic assumptions (but with two different approximations of the contact 


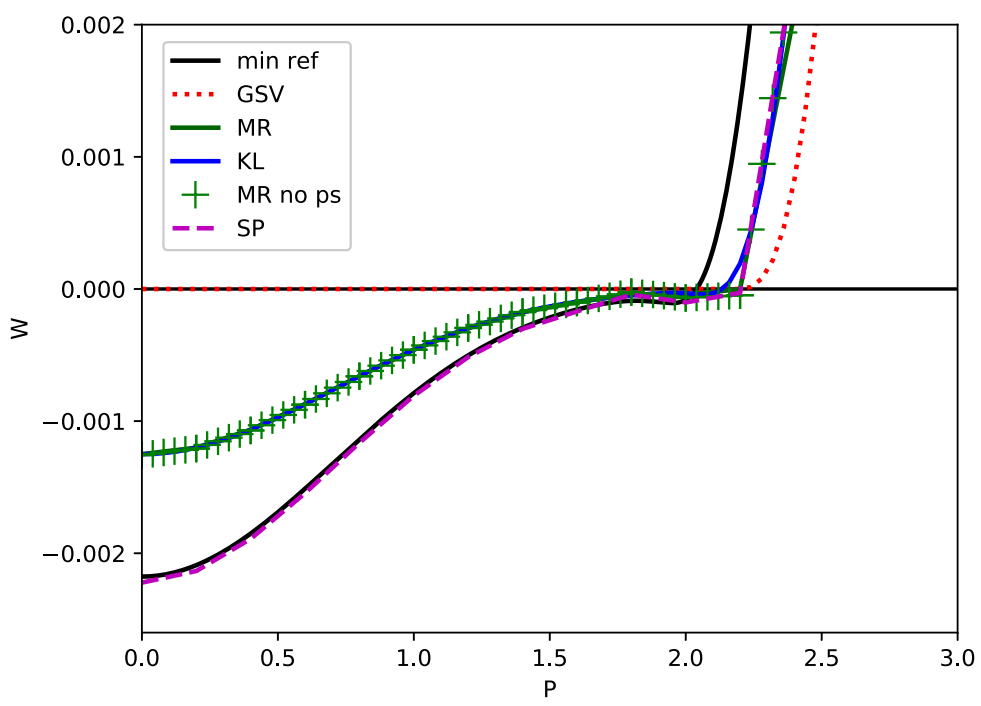

FIGURE 11. Upper-plane displacement on lateral edge.

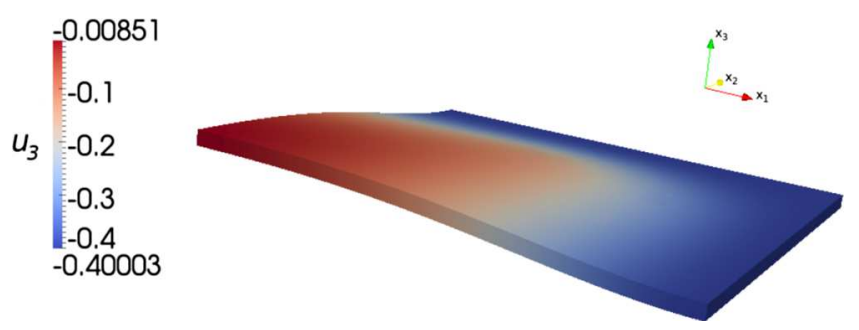

FIGURE 12. Thicker square plate $(\varepsilon=0.5 \mathrm{~m})$ on a paraboloid obstacle: 3D reference solution with the augmented Lagrangian method and $Q_{2}$-Lagrange elements.

condition), and the "SP" model is still the one offering better accuracy for fine meshes although the plate pinch is a priori limited for this case.

\section{Nitsche's PARAmeter Setting in the SOlid Plate CASE}

A very large value of Nitsche's parameter can deteriorate the solvability of the numerical approximation, especially when a Newton method is used. Conversely, at least for $\mathcal{O} \neq-1$, a too small value of Nitsche's parameter will not ensure the coercivity of formulation (2.11) and may lead to non-existence or even spurious solutions (see [10]). In this section, we present a mathematical justification of the choice of a Nitsche parameter $r$ close to $\frac{E}{\epsilon}$ as we used it in numerical tests. For the sake of simplicity, this justification is produced only for the solid plate model (although it can be adapted to other models) and for Tresca's friction instead of Coulomb's friction. Tresca's friction is a simplification of Coulomb's friction in which the friction threshold is assumed to be known and does not depend on the normal stress.

The following results give an estimate of the optimal Nitsche's parameter that ensures the coercivity of formulation (2.11) in the solid plate case. 


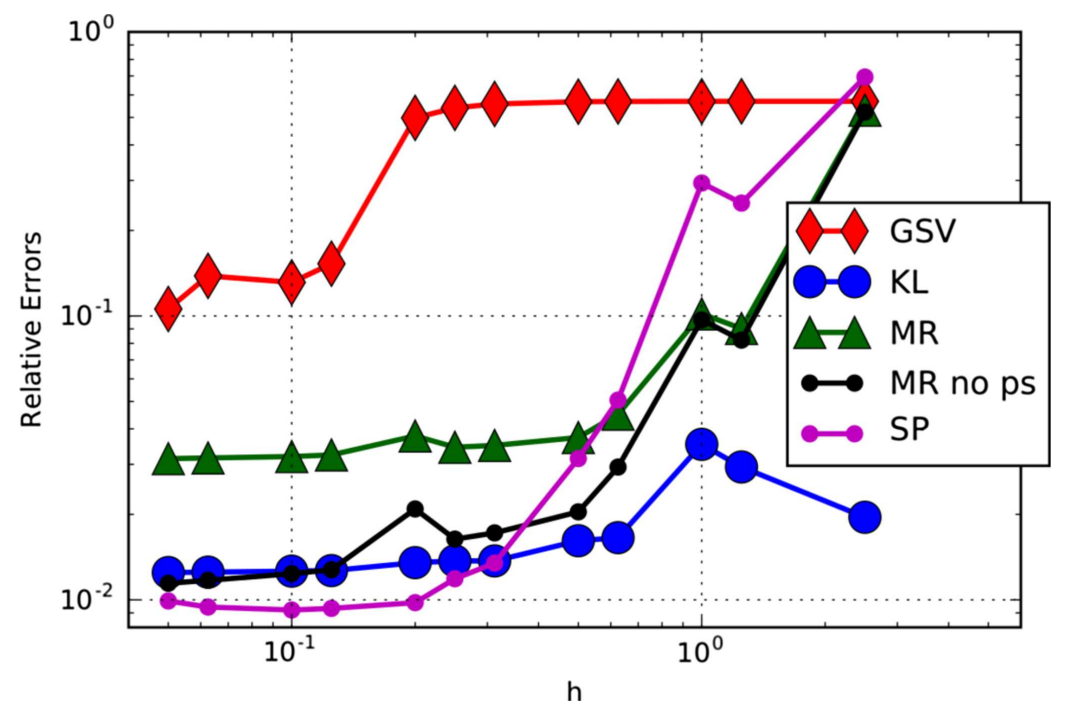

FiguRE 13. Relative $L^{2}\left(\Omega^{\varepsilon}\right)$-norm of the error in displacement with respect to the mesh size for the different plate models, comparatively to the 3D solution. Thicker plate on a paraboloid obstacle with no volume load.

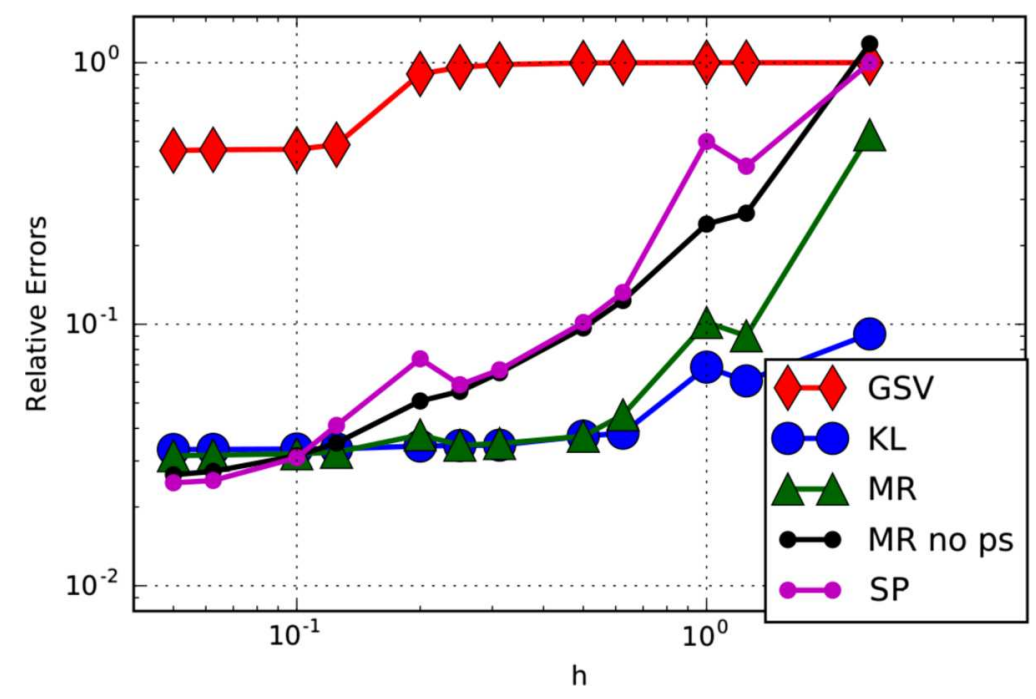

FiguRE 14. Relative $H^{1}\left(\Omega^{\varepsilon}\right)$-semi-norm of the error in displacement with respect to the mesh size for the different plate models, comparatively to the $3 \mathrm{D}$ solution. Thicker plate on a paraboloid obstacle with no volume load.

Proposition 7.1. For the solid plate model of Section 4.2 and for special case $\bar{n}=n=-e_{3}$ and with Tresca's friction instead of Coulomb's friction, a Nitsche's parameter value

$$
r>\zeta(\nu, \mathcal{O}) \frac{E}{\varepsilon}, \quad \text { with } \quad \zeta(\nu, \mathcal{O}):=\frac{(1+\mathcal{O})^{2}}{4(1+\nu)} \max \left(1, \frac{1}{2(1-2 \nu)}\right)
$$


ensures the monotonicity of the weak form (2.11) with kinematic assumptions (1.1) and consequently the existence and uniqueness of a solution.

Remark 7.2. The coefficient $\zeta(\nu, \mathcal{O})$ is close to unity for $\mathcal{O}=1$ and a Poisson ratio $\nu=1 / 3$. It goes to infinity in the incompressible limit.

Proof. The monotonicity of (2.11) for Tresca friction with a friction threshold $\chi$ (see [8] for Nitsche's method for Tresca friction) is ensured with kinematic assumptions (1.1) whenever for two arbitrary displacements $u^{1}, u^{2} \in \mathbb{V}^{\varepsilon}$ satisfying the kinematic assumptions (1.1) and $w=u^{1}-u^{2}$ one has

$$
\left\{\begin{array}{l}
\int_{\Omega^{\varepsilon}} \sigma(w): \gamma(w) \mathrm{d} \Omega \\
+\left.\frac{1}{r} \int_{\Omega}\left(\left[\sigma_{n}\left(u^{1}\right)+r\left(g-u_{n}^{1}\right)\right]_{-}-\left[\sigma_{n}\left(u^{2}\right)+r\left(g-u_{n}^{2}\right)\right]_{-}\right)\left(r\left(w_{n}\right)-\mathcal{O} \sigma_{n}(w)\right)\right|_{x_{3}=-\varepsilon} \mathrm{d} \Omega \\
-\left.\frac{1}{r} \int_{\Omega}\left(\mathbf{P}_{B(0, \chi)}\left(\sigma_{t}\left(u^{1}\right)-r u_{t}^{1}\right)-\mathbf{P}_{B(0, \chi)}\left(\sigma_{t}\left(u^{2}\right)-r u_{t}^{2}\right)\right) \cdot\left(r\left(w_{t}\right)-\mathcal{O} \sigma_{t}(w)\right)\right|_{x_{3}=-\varepsilon} \mathrm{d} \Omega \\
-\left.\frac{\mathcal{O}}{r} \int_{\Omega}|\sigma(w) n|^{2}\right|_{x_{3}=-\varepsilon} \mathrm{d} \Omega \geq \alpha\left\|u^{1}-u^{2}\right\|_{1, \Omega^{\varepsilon}}^{2}
\end{array}\right.
$$

Using as in ([8], Thm. 3.3) the monotonicity of the projections and Schwarz's inequality, we obtain the sufficient condition

$$
\left.\int_{\Omega^{\varepsilon}} \sigma(w): \gamma(w) \mathrm{d} \Omega-\frac{(1+\mathcal{O})^{2}}{4 r} \int_{\Omega} \mid \sigma(w) n\right)\left.\left.\right|^{2}\right|_{x_{3}=-\varepsilon} \mathrm{d} \Omega \geq \alpha\|w\|_{1, \Omega^{\varepsilon}}^{2} .
$$

The boundary $\Gamma_{D}^{\varepsilon}$ being assumed to be of non-zero measure and using Korn's inequality (see [10]), the monotonicity is ensured for $r>r_{0}$ and $r_{0}$ satisfying

$$
\int_{-\varepsilon}^{\varepsilon}(\sigma(w): \gamma(w)) \mathrm{d} x_{3}-\left.\frac{(1+\mathcal{O})^{2}}{4 r_{0}}|\sigma(w) n|^{2}\right|_{x_{3}=-\varepsilon} \geq 0 \quad \text { a.e. in } \Omega .
$$

Now, with the expressions obtained in Section 4.2, one obtains

$$
\begin{aligned}
\int_{-\varepsilon}^{\varepsilon}(\sigma(w): \gamma(w)) \mathrm{d} x_{3}= & \frac{2 \varepsilon E}{1+\nu} \gamma_{\alpha \beta}(\underline{w}) \gamma_{\alpha \beta}(\underline{w})+\frac{2 \varepsilon E \nu}{(1+\nu)(1-2 \nu)}\left(\gamma_{\eta \eta}(\underline{w})+\theta_{3}\right)^{2} \\
& +\frac{2 \varepsilon^{3} E}{3(1+\nu)} \gamma_{\alpha \beta}(\theta) \gamma_{\alpha \beta}(\theta)+\frac{2 \varepsilon^{3} E \nu}{3(1+\nu)(1-2 \nu)}\left(\gamma_{\eta \eta}(\theta)\right)^{2} \\
& +\frac{\varepsilon E}{1+\nu}\left(\partial_{\beta} \underline{w}_{3}+\theta_{\beta}\right)\left(\partial_{\beta} \underline{w}_{3}+\theta_{\beta}\right)+\frac{\varepsilon^{3} E}{3(1+\nu)}\left(\partial_{\beta} \theta_{3}\right)\left(\partial_{\beta} \theta_{3}\right) \\
& +\frac{2 \varepsilon E}{1+\nu} \theta_{3}^{2} \\
\left.|\sigma(w) n|^{2}\right|_{x_{3}=-\varepsilon}= & \frac{E^{2}}{(1+\nu)^{2}}\left(\theta_{3}+\frac{\nu}{1-2 \nu}\left(\gamma_{\eta \eta}(\underline{w})-\varepsilon \gamma_{\eta \eta}(\theta)+\theta_{3}\right)\right)^{2} \\
& +\frac{1}{4} \frac{E^{2}}{(1+\nu)^{2}}\left(\partial_{\beta} \underline{w}_{3}-\varepsilon \partial_{\beta} \theta_{3}+\theta_{\beta}\right)\left(\partial_{\beta} \underline{w}_{3}-\varepsilon \partial_{\beta} \theta_{3}+\theta_{\beta}\right) .
\end{aligned}
$$

Using Young's inequality, we write for $\delta_{1}>0$

$$
\begin{aligned}
& \left(\theta_{3}+\frac{\nu}{1-2 \nu}\left(\gamma_{\eta \eta}(\underline{w})-\varepsilon \gamma_{\eta \eta}(\theta)+\theta_{3}\right)\right)^{2} \\
& \quad \leq\left(1+2 \delta_{1}\right) \theta_{3}^{2}+\left(2+\frac{1}{\delta_{1}}\right) \frac{\nu^{2}}{(1-2 \nu)^{2}}\left(\left(\gamma_{\eta \eta}(\underline{w})+\theta_{3}\right)^{2}+\varepsilon^{2}\left(\gamma_{\eta \eta}(\theta)\right)^{2}\right)
\end{aligned}
$$


and for $\delta_{2}>0$

$$
\begin{aligned}
& \left(\partial_{\beta} \underline{w}_{3}-\varepsilon \partial_{\beta} \theta_{3}+\theta_{\beta}\right)\left(\partial_{\beta} \underline{w}_{3}-\varepsilon \partial_{\beta} \theta_{3}+\theta_{\beta}\right) \\
& \quad \leq\left(1+\delta_{2}\right)\left(\partial_{\beta} \underline{w}_{3}+\theta_{\beta}\right)\left(\partial_{\beta} \underline{w}_{3}+\theta_{\beta}\right)+\left(1+\frac{1}{\delta_{2}}\right) \varepsilon^{2} \partial_{\beta} \theta_{3} \partial_{\beta} \theta_{3} .
\end{aligned}
$$

Then, inequality (7.2) is satisfied for $r_{0}$ verifying

$$
\begin{aligned}
& r_{0} \geq \frac{(1+\mathcal{O})^{2}}{4}\left(1+2 \delta_{1}\right) \frac{E}{2(1+\nu) \varepsilon}, \quad r_{0} \geq \frac{(1+\mathcal{O})^{2}}{4}\left(2+\frac{1}{\delta_{1}}\right) \frac{E \nu}{2(1+\nu)(1-2 \nu) \varepsilon}, \\
& r_{0} \geq \frac{(1+\mathcal{O})^{2}}{4}\left(1+\delta_{2}\right) \frac{E}{4(1+\nu) \varepsilon} \quad \text { and } \quad r_{0} \geq \frac{(1+\mathcal{O})^{2}}{4}\left(1+\frac{1}{\delta_{2}}\right) \frac{3 E}{4(1+\nu) \varepsilon},
\end{aligned}
$$

which gives the announced result for $\delta_{1}=\frac{\nu}{1-2 \nu}$ and $\delta_{2}=3$. The existence and uniqueness of a solution can be deduced with the same arguments as in [10].

Note that the monotonicity of Tresca's friction law is crucial to prove the previous result. Its adaptation to Coulomb's friction remains an open problem.

\section{Conclusions}

We presented a systematic way to derive Nitsche's formulations for different kind of kinematics of plate models and studied these approaches in the context of finite element discretization. Several numerical studies were performed to illustrate the behavior of the proposed models. The numerical results allow us to draw the following main conclusions:

- For standard Kirchhoff-Love and Mindlin-Reissner plate models, which include a plane stress assumptions, the application of Nitsche's methods, as we propose in Section 3, simply leads to a penalized contact condition (although it is not the case for Gustafsson-Stenberg-Videman approach).

- The Gustafsson-Stenberg-Videman approach developed in [24] for Kirchhoff-Love plate model leads to a stricter non-interpenetration condition when its penalty coefficient $k$ is set to 0 .

- The numerical comparison with a fully 3D solution give a clear advantage to the penalized contact condition compared to a strict non-interpenetration condition for standard plate models. The presented Nitsche's approach for the Kirchhoff-Love and Mindlin-Reissner plate models can then be considered as a kind of justification of the use of a penalized contact condition for plate models since it allows to recover a certain elasticity in the transverse direction for the use of a convenient penalty coefficient $(r=E / \varepsilon)$. Of course, The Gustafsson-Stenberg-Videman approach with a similar penalty coefficient would give some similar results.

- We proposed a solid plate model consisting in a kinematics enriched by a pinching component combined with Nitsche's method for the contact condition. It combines the advantages of a good approximation and a stricter non-interpenetration condition on the contact surface. This model seems numerically to be richer for capturing the effects of contact stresses in the thickness of plate and reproduce the real 3D behavior of the structure in contact with an obstacle.

Acknowledgements. The work presented in this paper has been carried out with the generous support of ESI Group.

\section{REFERENCES}

[1] P. Alart and A. Curnier, A generalized Newton method for contact problems with friction. J. Méc. Théor. Appl. 7 (1988) 67-82.

[2] C. Annavarapu, M. Hautefeuille and J.E. Dolbow, A robust Nitsche's formulation for interface problems. Comput. Methods Appl. Mech. Eng. 225-228 (2012) 44-54. 
[3] K.J. Bathe and F. Brezzi, On the convergence of a four-node plate bending element based on Mindlin-Reissner plate theory and mixed interpolation. In: Vol. 21 of Proc. Conference on Mathematics of Finite Elements and Applications-V. Academic Press, New York (1985) 491-503.

[4] K.J. Bathe and E. Dvorkin, A four node plate bending element based on Mindlin-Reissner plate theory and mixed interpolation. Int. J. Numer. Methods Eng. 21 (1985) 367-383.

[5] J.L. Batoz and G. Dhatt, Modélisation des Structures par Éléments Finis, Poutres et plaques. Hermès Science Publications, Paris 2 (1990).

[6] S.C. Brenner, L.Y. Sung, H. Zhang and Y. Zhang, A quadratic $C^{0}$ interior penalty method for the displacement obstacle problem of clamped Kirchhoff plates. SIAM J. Numer. Anal. 50 (2012) 3329-3350.

[7] N. Büchter, E. Ramm and D. Roehl, Three-dimensional extension of non-linear shell formulation based on the enhanced assumed strain concept. Int. J. Numer. Methods Eng. 37 (1994) 2551-2568.

[8] F. Chouly, An adaptation of Nitsche's method to the Tresca friction problem. J. Math. Anal. Appl. 411 (2014) 329-339.

[9] F. Chouly and P. Hild, A Nitsche-based method for unilateral contact problems: numerical analysis. SIAM J. Numer. Anal. 51 (2013) 1295-1307.

[10] F. Chouly, P. Hild and Y. Renard, Symmetric and non-symmetric variants of Nitsche's method for contact problems in elasticity: theory and numerical experiments. Math. Comput. 84 (2015) 1089-1112.

[11] F. Chouly, R. Mlika and Y. Renard, An unbiased Nitsche's formulation of large deformation frictional contact and self-contact. Comput. Methods Appl. Mech. Eng. 325 (2017) 265-288.

[12] F. Chouly, M. Fabre, P. Hild, J. Pousin and Y. Renard, An overview of recent results on Nitsche's method for contact problems. In: Vol. 121 of Lecture Notes in Computational Science and Engineering. Geometrically Unfitted Finite Element Methods and Applications. Springer (2018) 93-141.

[13] F. Chouly, R. Mlika and Y. Renard, An unbiased Nitsche's approximation of the frictional contact between two elastic structures. Numer. Math. 139 (2018) 593-631.

[14] P.G. Ciarlet, The Finite Element Method for Elliptic Problems. North-Holland (1978).

[15] P.G. Ciarlet and P. Destuynder, A justification of two-dimensional linear plate model. J. Méc. 18 (1979) 315-343.

[16] A. Curnier, Q.C. He and A. Klarbring, Continuum mechanics modelling of large deformation contact with friction. In: Contact Mechanics. Springer (1995) 145-158.

[17] P. Destuynder, Mathematical Analysis of Thin Plate Models. Springer (1996).

[18] G. Drouet and P. Hild, Optimal convergence for discrete variational inequalities modelling Signorini contact in 2D and 3D without additional assumptions on the unknown contact set. SIAM J. Numer. Anal. 53 (2015) 1488-1507.

[19] C. Eck and J. Jarusek, Existence results for the static contact problem with Coulomb friction. Math. Models Methods Appl. Sci. 8 (1998) 445-468.

[20] M. Fabre, J. Pousin and Y. Renard, A fictitious domain method for frictionless contact problems in elasticity using Nitsche's method. SMAI J. Comput. Math. 2 (2016) 19-50.

[21] G. Fichera, Problemi elastostatici con vincoli unilaterali: il problema di Signorini con ambigue condizioni al contorno. Atti Accad. Naz. Lincei Mem. Cl. Sci. Fis. Mat. Natur. Sez. I 8 (1963) 91-140.

[22] A. Fritz, S. Hüeber and B.I. Wohlmuth, A comparison of mortar and Nitsche techniques for linear elasticity. Calcolo 41 (2004) $115-137$.

[23] T. Gustafsson, Finite element methods for contact problems, Ph.D. thesis. Aalto University publication series doctoral dissertations (2018).

[24] T. Gustafsson, R. Stenberg and J. Videman, A stabilised finite element method for the plate obstacle problem. BIT Numer. Math. 59 (2018) 97-124.

[25] R. Hauptmann and K. Schweizerhof, A systematic development of "solid-shell" element formulations for linear and non-linear analyses employing only displacement degrees of freedom. Int. J. Numer. Methods Eng. 42 (1998) 49-69.

[26] J. Jarusek, Contact problems with bounded friction, Coercive case. Czech. Math. J. 33 (1983) 237-261.

[27] M. Juntunen and R. Stenberg, Nitsche's method for general boundary conditions. Math. Comput. 78 (2009) $1353-1374$.

[28] J. Necas, J. Jarusek and J. Haslinger, On the solution of the variational inequality to the Signorini problem with small friction. Boll. Unione Mat. Ital. 17-B (1980) 796-811.

[29] J. Nitsche, Über ein variationsprinzip zur lösung von Dirichlet-problemen bei verwendung von teilräumen, die keinen randbedingungen unterworfen sind. Abh. Math. Semin. Univ. Hamburg 36 (1971) 9-15.

[30] K. Poulios and Y. Renard, An unconstrained integral approximation of large sliding frictional contact between deformable solids. Comput. Struct. 153 (2015) 75-90.

[31] C. Pozzolini and A. Léger, A stability result concerning the obstacle problem for a plate. J. Math. Pures Appl. 90 (2008) $505-519$.

[32] C. Pozzolini, Y. Renard and M. Salaun, Vibro-impact of a plate on rigid obstacles: existence theorem, convergence of a scheme and numerical simulations. IMA. J. Numer. Anal. 33 (2013) 261-294.

[33] Y. Renard, Generalized Newton's methods for the approximation and resolution of frictional contact problems in elasticity. Comput. Methods Appl. Mech. Eng. 256 (2013) 38-55.

[34] Y. Renard and K. Poulios, Automated FE modeling of multiphysics problems based on a generic weak form language. Submitted (2020).

[35] B.I. Wohlmuth, A mortar finite element method using dual spaces for the lagrange multiplier. SIAM J. Numer. Anal. 38 (2000) 989-1012.

[36] P. Wriggers and G. Zavarise, A formulation for frictionless contact problems using a weak form introduced by Nitsche. Comput. Mech. 41 (2008) 407-420. 